LPTHE 00-18

May 2000

\title{
On the Consistency of the Exact Renormalization Group Approach Applied to Gauge Theories in Algebraic Non-Covariant Gauges
}

\author{
M. Simionato \\ LPTHE, Université Pierre et Marie Curie (Paris VI) et Denis Diderot (Paris \\ VII), Tour 16, $1^{\text {er }}$ étage, 4, Place Jussieu, 75252 Paris, Cedex 05, France and \\ Istituto Nazionale di Fisica Nucleare (INFN) Frascati, Italy \\ E-mail: micheles@lpthe.jussieu.fr
}

\begin{abstract}
We study a class of Wilsonian formulations of non-Abelian gauge theories in algebraic non-covariant gauges where the Wilsonian infrared cutoff $\Lambda$ is inserted as a mass term for the propagating fields. In this way the Ward-Takahashi identities are preserved to all scales. Nevertheless BRST-invariance in broken and the theory is gauge-dependent and unphysical at $\Lambda \neq 0$. Then we discuss the infrared limit $\Lambda \rightarrow 0$. We show that the singularities of the axial gauge choice are avoided in planar gauge and light-cone gauge. In addition the issue of onshell divergences is addressed in some explicit example. Finally the rectangular Wilson loop of size $2 L \times 2 T$ is evaluated at lowest order in perturbation theory and a noncommutativity between the limits $\Lambda \rightarrow 0$ and $T \rightarrow \infty$ is pointed out.
\end{abstract}

PACS: 11.10.Hi, 11.15.-q, 11.38.Bx.

Keywords: Renormalization Group, Axial gauge, Planar gauge, Lightcone gauge, Gauge dependence 


\section{Introduction}

In recent years, a lot of activity has been devoted to the study of Quantum Field Theory in the framework of the Exact Renormalization Group Equation (ERGE) of Wilson [1]. The principal advantage of this approach is the fact that the ERGE can be solved numerically at the non-perturbative level: this feature in principle pave the way to a number of important applications. However, in practice, the method works well for scalar theories, where there is an extensive literature and a collection of reliable numerical results (see [2] for a recent review and a complete bibliography) whereas its application to gauge theories should be regarded as problematic. The root of the difficulties becomes clear if we remind the logic of the ERGE approach in the formalism of the one-particle irriducible (1PI) effective action [3, 4], which is the following: i) take a given Quantum Field Theory and introduce a fictitious infrared cutoff $\Lambda$ by modifing the bare propagator in some way; ii) write the functional identity which describes how the 1PI effective action of the theory changes when the infrared cutoff is lowered; iii) fix the boundary conditions at the ultraviolet scale $\Lambda_{0}$ by identifying the effective action with the local bare action and integrate down numerically [3] or analytically in perturbation theory [4] the equation up to the the infrared value $\Lambda \ll \Lambda_{0}$.

This logic is perfectly legitimate in scalar theories, but quite delicate in the case of gauge theories since there is no way of inserting the infrared cutoff in a way consistent with gauge-invariance (we mean with BRST-invariance): as a consequence the $\Lambda \neq 0$ theory is gauge-dependent and unphysical and the limit $\Lambda \rightarrow 0$ must be taken strictly. This generates two distinct problems.

The first problem, which has been extensively studied in the literature [5, 6], is the following. Since the infrared cutoff breaks BRST-invariance, the usual Slavnov-Taylor identities have to be modified and assume a very complicate form [5] such that it is practically impossible to find truncations of the effective action consistent with the modified identities. As a consequence at the non-perturbative level there is no rigorous control on the fact that the gauge-invariant theory is recovered when the infrared cutoff is removed. On the contrary at the perturbative level this is guaranteed by a theorem: no matter how the infrared cutoff is inserted, the $\Lambda \rightarrow 0$ theory is gauge invariant provided that we choose the ultraviolet bare action in a very precise way, which can be established in perturbation theory to all orders by solving the fine-tuning equations discussed in [6]. Therefore, at least in principle we could consider solved this problem, even if in practice the fine-tuning equations are 
quite involved and cannot be solved out of perturbation theory.

The second problem, which in our knowledge has been treated only partially in the literature, is the question of the regularity of the $\Lambda \rightarrow 0$ limit. Actually the smoothness of this limit is guaranteed only for the proper vertices at non-exceptional configuration of momenta [7]: but these are not physical quantities. On the contrary, the limit is extremely delicate in the computation of physical quantities as for instance the interquark potential: we will see in this paper that the limit must be taken with great care in order to have sensible results. In such a situation, a naive numerical analysis of the problem is certainly questionable. Apparently, this subtle point has never been recognized in the previous literature and is explicitly analyzed here for the first time.

The guideline of our research program consists in setting up a formalism where these problems can be faced analytically with perturbative computations, in such a way to have definite answers on the recovering of the gauge invariance and on the regularity or the singularity of the $\Lambda \rightarrow 0$ limit for physical quantities. In this logic, in a couple of recent papers [8, 9] we presented a Wilsonian formulation of gauge theories in which the Wilsonian infrared cutoff $\Lambda$ is introduced as a mass-like term for the propagating fields. In this way the Wilson's Renormalization Group Equation for the 1PI effective action becomes consistent with the Ward-Takahashi identities to all scales. This important property, which allows to solve the problem of finding gauge-consistent truncations but not the question on the regularity of the $\Lambda \rightarrow 0$ limit, was proved in the Abelian case in covariant gauges \& and in the non-Abelian case in algebraic non-covariant gauges [9]. In this latter case, the crucial point was the fact that in algebraic non-covariant gauges the ghost fields decouple and the gauge-symmetry can be implemented via simple Ward-Takahashi identities instead that in terms of complicated SlavnovTaylor identities. However, as we said in [9], even if the implementation of the gauge symmetry is quite efficient in these formulations there are various disadvantages to be taken in account. The first disadvantage, common also to the covariant version of the method, is the need for an explicit regularization of the evolution equation, since when the mass cutoff is employed the ultraviolet momenta are not sufficiently suppressed. However, in our view this is a little price to pay since, at least in perturbation theory, there exist gauge-invariant techniques to manage the ultraviolet problem (dimensional regularization, higher derivative regularization, etc.). The second disadvantage is the gauge-dependence of the would-be physical quantities at $\Lambda \neq 0$. 
However, even this problem is not so dramatic since the gauge-dependence can be controled throught the generalized functional identities described in [9]. It is only the third disadvantage, i.e. the fact that non-covariant gauges have a very delicate $\Lambda \rightarrow 0$ limit, which is a very serious one. This feature gives rise to difficulties since if the $\Lambda \rightarrow 0$ limit does not exist for quantities which are instead well defined in other gauges the whole approach should be considered as suspicious. In practice this fact does not prevent phenomenological applications since there are infrared safe quantities which can be computed in some approximation (i.e. by a truncation of the evolution equation) without encountering problems. Nevertheless in questions of principle one would like to have a formalism with a well definite perturbative expansion and fully consistent with other gauges for any physical quantity. This is why in this paper we carefully study the infrared limit of the nonAbelian theory in different algebraic non-covariant gauges. We will see in particular that the planar gauge and the light-cone gauge are regular in the $\Lambda \rightarrow 0$ limit whereas the axial gauge is singular. The origin of the problems of the axial gauge will be connected with the spurious infrared divergences introduced by the double pole term in the propagator, i.e. the term proportional to $n^{2} q_{\mu} q_{\nu} /[n \cdot q]^{2}$. This is hardly unexpected, since it is well known that in the standard approach using the Cauchy Principal Value (CPV) prescription the singularities connected with the double pole are responsible for many serious problems, as the loss of unitarity $\| 10$ and the failure of the Wilson loop consistency check [11]. In particular this latter quantity is the most interesting to study since it is connected with the interquark potential, which is the typical quantity one would like to compute non-perturbatively in the Wilson renormalization group approach [12]. We recall that problems are expected to be present in the perturbative computation of this quantity since it was shown long time ago, working in the temporal axial gauge [13 that the Wilson loop of sides $2 L \times 2 T$ which directly corresponds to the interquark potential computed at order $g^{4}$ is different from the corresponding computation in covariant gauges. More dramatically, in the standard perturbative expansion, the temporal gauge Wilson loop does not exponentiate in the limit $T \rightarrow \infty$ i.e. it is not of the form

$$
W_{\Gamma_{L T}} \stackrel{T \rightarrow \infty}{=} \exp [-i 2 T V(2 L)]
$$

(see [14] for a critical analysis). The same problem was found in the spatial axial gauge for loops in the Euclidean space with the side $T$ in the direction 
of the axial vector $n^{\mu}$ [ [11]. The origin of the problem was found in the Cauchy Principal Value Prescription which in fact breaks gauge-invariance. There has been a lot of work in the literature to try to solve this problem (see the references cited in the monograph [15]). In the Wilsonian approach one naturally obtains a regularization of spurious divergences different from the CPV prescription and which is consistent with unitarity. Nevertheless, as we anticipated in [9], still one expects to have problems in the infrared limit $\Lambda \rightarrow 0$. These problems are discussed in detail in this paper: in addition we prove that they are absent in more stable algebraic non-covariant gauges, as the planar gauge and the light-cone gauge. This is not a surprise since it is well known in the literature that these gauge are safe with traditional prescriptions (the CPV for the planar gauge and the Mandelstam-Leibbrandt (ML) for the light-cone gauge) and in particular the Wilson loop consistency check is successfully passed [18, 19] at least at order $O\left(g^{4}\right)$ in perturbation theory. The light-cone gauge in particular should be considered as a promising starting point for our analysis. However we stress that to prove that the Wilsonian i.e. massive formulation tends to the standard massless formulation (which is safe) in the $\Lambda \rightarrow 0$ limit is a subtle point since there are infrared sensitive quantities where the massive version and the massless version of the same gauge choice are different, i.e. the $\Lambda \rightarrow 0$ limit is singular. The non-trivial point is to show that this does not happen for physical quantities. In principle this could also be false in a finite order perturbative computation. As we said, one of the motivation of this paper it to set up a workable formalism where such a question can be answered with a reasonable analytic effort.

In order to compare and contrast with the previous Wilsonian literature, we remind that a Wilsonian approach to algebraic non-covariant gauges has already been presented in [16, 17]; nevertheless our analysis differs from that approach, where a generic cutoff function is employed thus totally obscuring a number of important properties and difficulties of the formalism: in particular the gauge-invariance issue and the infrared problems can be clearly studied only within our framework. In spite of the peculiarities of our approach, we stress that our results should be regarded as quite general, since there is a large class of cutoff functions which in the infrared behave as a mass term: for instance the most used cutoff in the numerical ERGE literature [3]

\footnotetext{
${ }^{1}$ Obviously there exist loops which give the same result in axial gauge and in Feynman gauge and in general smooth loops are expected to be safe.
} 
the exponential cutoff

$$
R_{\Lambda}(q)=\frac{q^{2} \exp \left(-q^{2} / \Lambda^{2}\right)}{1-\exp \left(-q^{2} / \Lambda^{2}\right)}
$$

is in this class since $R_{\Lambda}(q) \simeq \Lambda^{2}$ for $q^{2} \ll \Lambda^{2}$ and thus soft momenta are effectively screened as if there were a mass $\Lambda^{2}$. This is not, however, the general case, since for instance the sharp cutoff is not in this class't. In general we expect that the situation of the infrared limit for other cutoff functions is worse than in the mass-cutoff case, since the gauge-symmetry is completely broken, Ward-Takahashi identities have a cumbersome form, and possible gauge-cancellations between different divergent contributions are lost. Actually one could speculate that at the non-perturbative level, due to infinite resummations of classes of Feynman diagram the infrared problem could be eventually solved. However, as a matter of fact, the perturbative expansion is the only controled analytical tool we have at our disposition. This is why we are interested in giving a Wilsonian formulation in which i) a well defined perturbative expansion can be defined and ii) it is possible to perform practical, analytical computations with an effort not too much bigger than in the standard massless approach. In particular in this work we discuss a general method and a series of tricks to analytically compute finite parts of one-loop Feynman diagrams in the $\Lambda \rightarrow 0$ limit. This is relevant in preparation to a computation of the Wilson loop up to order $O\left(g^{4}\right)$.

The plan of the paper is the following: in section 2 we review the basics of the standard gauge-fixing procedure, pointing out some subtle aspects which are usually not noticed since an infrared regularization consistent with BRST symmetry is assumed, which is not our case. In section 3 we study the properties of the general linear gauge $L^{\mu}(p) A_{\mu}^{a}(p)=0$ in presence of a masslike cutoff. In particular the massive axial gauge, the massive planar gauge and the massive light-cone gauge are analyzed. In section 4 we discuss the problems of the axial gauge as connected to the presence of the double pole in the propagator, whereas in sections 5 and 6 we show that these problems

2 This can be seen for instance by using the approximation to the sharp cutoff

$$
R_{\Lambda}(q)=\frac{q^{2} \exp \left(-\left(q^{2} / \Lambda^{2}\right)^{b}\right)}{1-\exp \left(-\left(q^{2} / \Lambda^{2}\right)^{b}\right)}
$$

with $b \rightarrow \infty$. In this case the infrared modes are very strongly suppressed since $R_{\Lambda}(q)$ is divergent as $q^{2} \rightarrow 0$. The same is obtained with other regularizations of the sharp cutoff. 
are absent in planar and light-cone gauge. In section 7 the Wilson loop for a finite rectangular path is explicitly computed up to order $O\left(g^{2}\right)$ and it is shown that the physical limit $\Lambda \rightarrow 0$ exists and is independent of the gaugefixing choice. Section 8 resume our work and contains our conclusions. Two appendices close the paper: appendix $\mathrm{A}$ is a simple but instructive exercice that shows the gauge-dependence problem in the computation of a physical quantity, the pressure of a free photon gas; appendix B collects the technical tools and tricks which are used in the text to compute one-loop Feynman diagrams.

\section{Remarks on the gauge-fixing procedure}

In a strictly gauge-invariant theory one cannot define a gauge-field propagator since strict gauge-invariance implies that the free two-point function is transverse and therefore cannot be inverted. As a consequence the starting point of the perturbative analysis in terms of Feynman diagrams is missing. Thus, in order to define a perturbative quantum field theory from a classical gauge invariant field theory one is forced to break gauge invariance through the addition of a gauge-fixing term to the classical action?. The way gauge-invariance is broken depends on the chosen gauge-fixing term, which is at large extent arbitrary: the essential requirement is that the gauge-fixing term, depending on the gauge field and other fields (ghosts, antighosts and auxiliary fields), must be a BRST-cocycle: in this hypothesis it is possible to prove that the physical quantities are gauge-fixing independent and therefore that there are not physical arbitrarities.

In the path integral approach the gauge-fixing is usually introduced by means of the Faddeev-Popov argument: since there is an infinite number of gauge-equivalent configurations giving the same contribution to the functional integral the partition function is ill defined. Then the problem is solved by integrating only on representative of any gauge orbit. The representative

are fixed by means of a gauge fixing condition of kind $\mathcal{F}^{a}(A)=0$; typical

\footnotetext{
${ }^{3}$ Outside perturbation theory, for instance in the lattice approach, one can define the theory without an explicit gauge-fixing. However in order to show that the continuum limit exists, which has been rigorously proved only in perturbation theory, a gauge-fixing term is needed even in the lattice formulation. In general the gauge-fixing term is unavoidable to make contact with the perturbative formulation, which is the only one we analytically control.
} 
choices are $\mathcal{F}^{a}(A)=\partial^{\mu} A_{\mu}^{a}$ (covariant gauges) or $\mathcal{F}^{a}(A)=n^{\mu} A_{\mu}^{a}$ (algebraic non-covariant gauges). These latter are interesting even because they avoid the problem of Gribov copies, i.e. the choice of the representative of the gauge orbit is unique [15].

Now it is convenient to introduce a bit of notations (the full list of our notations is reported in [9]). A gauge transformations of parameters $\delta \omega=$ $\delta \omega \cdot T=\delta \omega^{a} T_{a}$ is denoted by

$$
\delta_{G} A_{\mu}=D_{\mu} \delta \omega, \quad \delta_{G} \psi=i g \delta \omega \psi, \quad \delta_{G} \bar{\psi}=-i g \bar{\psi} \delta \omega,
$$

where $T_{a}$ are the generators of $S U\left(N_{c}\right)$ in the fundamental representation and $N_{c}=3$ is the number of colors. The set of physical fields of theory is denoted by $\phi=\left(A_{\mu}^{a}(p), \psi_{i}(p), \bar{\psi}^{i}(p)\right)$ and the bare action with $S_{B}(\phi)$; the expression $\frac{\delta \mathcal{F}}{\delta \omega}$ denotes the differential operator $\frac{\delta \mathcal{F}}{\delta A_{\mu}} D_{\mu}$. A gauge-invariant ultraviolet regularization, which exists in non-chiral theories, is understood. With these notations the gauge-fixed partition function can be written as

$$
Z_{\mathcal{F}}=\int[d \phi] \operatorname{det} \frac{\delta \mathcal{F}}{\delta \omega} \delta(\mathcal{F}(A)) e^{i S_{B}(\phi)} .
$$

The usefulness of the gauge-fixed approach is based in the following fundamental property: if the observable $O(\phi)$ is gauge-invariant, then its mean value in the gauge $\mathcal{F}^{a}(A)=0$, defined as

$$
<O(\phi)>_{\mathcal{F}}=\frac{1}{Z_{\mathcal{F}}} \int[d \phi] \operatorname{det} \frac{\delta \mathcal{F}}{\delta \omega} \delta(\mathcal{F}(A)) e^{i S_{B}(\phi)} O(\phi),
$$

is independent of the gauge-fixing function $\mathcal{F}^{a}$ :

$$
\delta_{G} O(\phi)=0 \Rightarrow<O>_{\mathcal{F}}=<O>_{\mathcal{F}^{\prime}}, \quad \mathcal{F} \neq \mathcal{F}^{\prime} .
$$

This means that in the computation of physical quantities we have the freedom of choosing any possible gauge-fixing.

We think that it is useful to review here the formal argument that support this statement with the scope of showing where and why this argument fails in the Wilsonian formalism.

The first step consists in introducing anticommuting fields $C$ and $\bar{C}$ and commuting auxiliary fields $\lambda$ in order to rewrite the determinant $\operatorname{det} \frac{\delta \mathcal{F}}{\delta \omega}$ as a 
functional integral over Grassmannian variablest

$$
\operatorname{det} \frac{\delta \mathcal{F}}{\delta \omega} \propto \int[d C d \bar{C}] \exp \left[-i \bar{C} \cdot \frac{\delta \mathcal{F}}{\delta \omega} C\right]
$$

and the functional delta function as

$$
\delta(\mathcal{F}(A))=\int[d \lambda] \exp [i \lambda \cdot \mathcal{F}(A)]
$$

then the partition function (defined up to a multiplicative constant) can be rewritten in the form

$$
Z_{\mathcal{F}}=\int[d C d \bar{C} d \lambda d \phi] \exp \left[i S_{\mathcal{F}}(\phi, C, \bar{C}, \lambda)\right]
$$

where the total bare action

$$
S_{\mathcal{F}}=S_{B}+\lambda \cdot \mathcal{F}(A)-\bar{C} \cdot \frac{\partial \mathcal{F}}{\partial A_{\mu}} D_{\mu} C
$$

is invariant under the BRST-tranformation

$$
s A_{\mu}=D_{\mu} C, \quad s C=-\frac{1}{2} g C \times C, \quad s \bar{C}=\lambda, \quad s \lambda=0
$$

for any choice of the gauge-fixing function. The cohomological property of the BRST tranformation $s^{2}=0$ allows us to prove the independence of BRSTinvariant quantities from the gauge-fixing choice. Consider for instance a class of gauge-fixing functions $\mathcal{F}_{\varepsilon}^{a}(A)$ determined by one or more continuous parameters $\varepsilon$ : then we have to prove that physical quantities are independent of $\varepsilon$. This can be easily shown by observing that the gauge-fixing term can be rewritten as a trivial cocicle

$$
\lambda \cdot \mathcal{F}_{\varepsilon}(A)-\bar{C} \cdot \frac{\delta \mathcal{F}_{\varepsilon}}{\delta A_{\mu}} D_{\mu} C=s\left(\bar{C} \cdot \mathcal{F}_{\varepsilon}(A)\right) .
$$

Using the fact that trivial cocycles do not contribute to the partition function, i.e.

$$
\int[d \Phi] e^{i S_{\mathcal{F}}(\Phi)} s f(\Phi) \equiv 0
$$

\footnotetext{
${ }^{4}$ Here we use the hermiticity conditions $C=C^{\dagger}$ and $\bar{C}=-\bar{C}^{\dagger}$ [21] such as the relation $\left(\bar{C} \cdot \frac{\delta \mathcal{F}}{\delta \omega} C\right)^{\dagger}=\bar{C} \cdot \frac{\delta \mathcal{F}}{\delta \omega} C$ holds.
} 
(we have denoted by $\Phi=(\phi, C, \bar{C}, \lambda)$ the full set of fields of the theory) one sees that the partition function is $\varepsilon$-independent

$$
\frac{\mathrm{d}}{\mathrm{d} \varepsilon} Z_{\varepsilon}=\int[d \Phi] e^{i S_{\mathcal{F}}(\Phi)} s\left(\bar{C} \cdot \frac{\mathrm{d}}{\mathrm{d} \varepsilon} \mathcal{F}_{\varepsilon}(A)\right)=0
$$

and the same holds for BRST-invariant observables $O(\Phi)$ such as $s O=0$ :

$$
\frac{\mathrm{d}}{\mathrm{d} \varepsilon}<O>_{\varepsilon}=\frac{1}{Z_{\varepsilon}} \int[d \Phi] e^{i S_{\mathcal{F}}(\Phi)} s\left(O(\Phi) \bar{C} \cdot \frac{\mathrm{d}}{\mathrm{d} \varepsilon} \mathcal{F}_{\varepsilon}(A)\right)=0
$$

Unfortunately this proof is purely formal: a rigorous analysis must face the question of infrared divergences which can invalidate the argument. In fact, the perturbative expansion has no meaning at all, if we do not specify carefully as the infrared divergences are managed. The need for an infrared regularization can conflict with BRST-invariance and in general to show that gauge-invariance is recovered when the infrared regulator is removed is nontrivial since the existence of the infrared limit for physical quantities is a very delicate point. To our knowledge this issue has been not enough emphasized in the literature on the Wilson Renormalization Group, in spite of the fact that it is a very crucial one. Actually, even if the recovering of the gauge symmetry at $\Lambda=0$ has been proved for proper vertices at non-exceptional configurations of momenta [7], there are no theorems guaranteeing the infrared safety of important physical quantities as the interquark potential, which is the first thing one would like to compute in the Wilson renormalization group approach [12. In particular, as we said in the introduction, there are problems in its perturbative computation. The reason is that the fundamental property of the exponentiation of the Wilson loop at $T \rightarrow \infty$ is lost at $\Lambda \neq 0$ since it is related to highly non-trivial gauge-cancellations between different contributions which are lost when BRST-invariance is broken by the infrared cutoff. In other terms we expect a non-commutativity of limits $T \rightarrow \infty$ and $\Lambda \rightarrow 0$. This can be seen even at $O\left(g^{2}\right)$ in perturbation theory using the non-covariant formalism and it will be discussed at length in section 7. This peculiarity suggests that numerical computations of the interquark potential should be performed with great care. 


\section{The general linear gauge}

In general we are interested in linear gauges where $\mathcal{F}(A)$ is a linear function of the gauge field,

$$
\mathcal{F}(A)=L^{\mu}(p) A_{\mu}(p), \quad L_{\mu}(p)=a n_{\mu}+b p_{\mu} .
$$

Notice that this is a class of interpolating gauges involving both the covariant gauges (if $a=0$ ) and the algebraic non-covariant gauges (if $b=0$ ). Other non-covariant gauges, as for instance the Coulomb one, can be obtained for particular values of $a$ and $b$. In order to integrate out the auxiliary fields it is convenient to introduce a small convergence factor in the functional integral measure by adding to the BRST action a term

$$
\int_{x} \frac{1}{2} \xi_{2} \lambda \cdot \lambda,
$$

where $\xi_{2}$ is a positive parameter of mass dimension -2 . This terms is a trivial cocycle and therefore does not change the essential properties of the system; in particular by using standard techniques [24] it is possibile to prove that physical quantities are formally independent of $\xi_{2}$. By using the linear equations of motion

$$
\lambda^{a}=-\frac{1}{\xi_{2}} L^{\mu} A_{\mu}^{a}
$$

we can eliminate the auxiliary fields $\lambda^{a}$ and consider the reduced action

$$
S_{\text {lin.g. }}^{\text {red }}=\int_{x}-\frac{1}{4} F_{\mu \nu} \cdot F^{\mu \nu}-\bar{C} \cdot L^{\mu} D_{\mu} C+\int_{x} \frac{1}{2 \xi_{2}} L^{\mu} A_{\mu} \cdot L^{\nu} A_{\nu} .
$$

To this reduced three-level action, we add an infrared cutoff as a mass-like term 9

$$
S_{\text {lin.g. }}^{\text {red }}(A, C, \bar{C}, \Lambda)=S_{\text {lin.g. }}^{\text {red }}(A, C, \bar{C})+\int_{x} \frac{1}{2} \Lambda^{2} A^{2} .
$$

Now we can define an invertible massive propagator for the gauge fields. The explicit form of the propagator can be obtained in Euclidean space (Euclidean notations are recalled in appendix B) by inverting the matrix

$$
D_{\Lambda, \mu \nu}^{-1}\left(p_{E}\right)=\left(t_{\mu \nu}\left(p_{E}\right) p_{E}^{2}+\frac{1}{\xi_{2}} L_{\mu}\left(p_{E}\right) L_{\nu}\left(p_{E}\right)+\Lambda^{2} \delta_{\mu \nu}\right),
$$


where $t_{\mu \nu}\left(p_{E}\right)$ denotes the transverse projector in Euclidean space

$$
t_{\mu \nu}\left(p_{E}\right)=\delta_{\mu \nu}-\frac{p_{E, \mu} p_{E, \nu}}{p_{E}^{2}}
$$

In particular we are interested in the $\xi_{2} \rightarrow 0$ limit, when one eigenvalue of the propagator vanishes, due to the transversality property

$$
L^{\mu} D_{\Lambda, \mu \nu}\left(p_{E}\right) \stackrel{\xi_{2} \rightarrow 0}{=} 0, \quad \forall \Lambda
$$

and therefore $D_{\Lambda, \mu \nu}$ is not invertible (for any $\Lambda$ ). The final result is

$$
\begin{aligned}
& D_{\Lambda, \mu \nu}\left(p_{E}\right) \stackrel{\xi_{2} \rightarrow 0}{=} \frac{1}{p_{E}^{2}+\Lambda^{2}} \delta_{\mu \nu}-\frac{p \cdot L\left(L_{\mu} p_{E, \nu}+L_{\nu} p_{E, \mu}\right)}{\left(p_{E}^{2}+\Lambda^{2}\right)\left(\left(p_{E} \cdot L\right)^{2}+L^{2} \Lambda^{2}\right)}+ \\
& \frac{p_{E, \mu} p_{E, \nu} L^{2}}{\left(p_{E}^{2}+\Lambda^{2}\right)\left(\left(p_{E} \cdot L\right)^{2}+L^{2} \Lambda^{2}\right)}-\frac{\Lambda^{2} L_{\mu} L_{\nu}}{\left(p_{E}^{2}+\Lambda^{2}\right)\left(\left(p_{E} \cdot L\right)^{2}+L^{2} \Lambda^{2}\right)} .
\end{aligned}
$$

Notice that in limit $\xi_{2} \rightarrow 0$ the propagator is invariant up to rescaling of the gauge-fixing $L\left(p_{E}\right) \rightarrow C\left(p_{E}^{2}\right) L\left(p_{E}\right)$. In particular for $L_{\mu} \propto n_{\mu}$ we recover the massive axial gauge introduced in [9] which satisfies simple Ward identities, whereas for $L_{\mu} \propto p_{\mu}$ we obtain a massive version of the usual Landau gauge, which does not satisfies linearly broken Ward identities, but instead satisfies a little modification of the usual Slavnov-Taylor identities (this is the Curci-Ferrari model in Landau gauge [20]). We have also computed the explicit form of the propagator at $\xi_{2} \neq 0$, but the resulting expression is not particularly illuminating and there is no scope in writing it here.

\subsection{Planar gauge}

The standard massless version of the planar gauge is formally obtained from the generalized axial gauge if we replace the parameter $\xi_{2}$ with the momentum dependent function $\xi_{2}(p)=n^{2} / p^{2}$; in this case the BRST action reads

$$
S_{\text {planar }}^{B R S T}=\int_{x}-\frac{1}{4} F_{\mu \nu} \cdot F^{\mu \nu}-\bar{C} \cdot n^{\mu} D_{\mu} C+\lambda \cdot n^{\mu} A_{\mu}+\frac{1}{2} \lambda \cdot \frac{n^{2}}{\partial^{2}} \lambda
$$

or, after elimination of the auxiliary fields,

$$
S_{\text {planar }}^{\text {red }}=\int_{x}-\frac{1}{4} F_{\mu \nu} \cdot F^{\mu \nu}-\bar{C} \cdot n^{\mu} D_{\mu} C-\frac{1}{2} n^{\mu} A_{\mu} \cdot \partial^{2} n^{\nu} A_{\nu}
$$


The planar gauge is less problematic than the axial gauge, but the price to pay is in a more complicate realization of symmetries: in opposition to the axial gauge case, the action (25) does not satisfies simple Ward-Takahashi identities (the reason being the presence of derivatives in the gauge-fixing term) but instead Slavnov-Taylor-like identities; moreover ghost fields play a non-trivial role even if less crucial than in covariant gauges 2 . The planar gauge has been studied quite intensively in the literature and has some interest in itself, and also in comparison with the axial gauge and the light-cone gauge in the problem of inconsistencies of perturbation theory. Moreover the computation of Feynman diagrams in planar gauge is similar but simpler than in light-cone gauge. For these reasons we will analyze in detail this gauge choice. We found that the more convenient way to insert the infrared cutoff in order to have a simple propagator is to modify the massless BRST action as

$$
\begin{aligned}
S_{\text {planar }}(\Lambda)= & \int_{x}-\frac{1}{4} F_{\mu \nu} \cdot F^{\mu \nu}-\bar{C} \cdot n^{\mu} D_{\mu} C+\lambda \cdot n^{\mu} A_{\mu} \\
& +\int_{x} \frac{1}{2} \Lambda^{2} A_{\mu} \cdot A^{\mu}+\frac{1}{2} \lambda \cdot \frac{n^{2}}{\partial^{2}+\Lambda^{2}} \lambda .
\end{aligned}
$$

This gives as tree level reduced action

$$
\begin{aligned}
S_{\text {planar }}^{\text {red }}(\Lambda)= & \int_{x}-\frac{1}{4} F_{\mu \nu} \cdot F^{\mu \nu}+\frac{1}{2} \Lambda^{2} A \cdot A+ \\
& -\frac{1}{2 n^{2}} n_{\mu} A^{\mu} \cdot\left(\partial^{2}+\Lambda^{2}\right) n_{\mu} A^{\mu}-\bar{C} \cdot n^{\mu} D_{\mu} C .
\end{aligned}
$$

Notice that the "mass" $\Lambda^{2}$ multiplies the term

$$
\frac{1}{2} A^{\mu}\left(g_{\mu \nu}-\frac{n_{\mu} n_{\nu}}{n^{2}}\right) A^{\nu}=\frac{1}{2} A^{i} g_{i j} A^{j}, \quad i, j \in\{0,1,2\}
$$

and thus only transverse (with respect to $n_{\mu}$ ) degrees of freedom are screened. There are other possible ways of introducing the infrared cutoff in the planar gauge, but this is the more interesting one in the sense that one obtains a

\footnotetext{
${ }^{5}$ For instance the planar gauge has the interesting feature that all diagrams involving ghost loops identically vanishes [15, 25]. This property is preserved even in the massive version. Nevertheless, there are non-vanishing vertex corrections including ghosts as external lines. In principle this could be avoided by considering a formulation without ghost fields [23] but then the Ward identities becomes quite cumbersome.
} 
propagator which is simple and very similar to the propagator of the lightcone gauge. Its explicit form in Minkowsky space is

$$
-D_{\Lambda, \mu \nu}(p)=\frac{1}{p^{2}-\Lambda^{2}+i \varepsilon}\left\{g_{\mu \nu}-\frac{n_{\mu} p_{\nu}+n_{\nu} p_{\mu}}{[p \cdot n]}+\frac{\Lambda^{2} n_{\mu} n_{\nu}}{[p \cdot n]^{2}}\right\} .
$$

The most important difference with respect to the massive axial gauge case is the fact that in the massive planar gauge there are spurious divergences at $p \cdot n=0$ even at $\Lambda \neq 0$. Therefore we need an explicit prescription to manage them. The simplest choice which works, at least up to the order $O\left(g^{4}\right)$ in the stardard massless case

$$
\frac{1}{[p \cdot n]} \equiv \lim _{\varepsilon \rightarrow 0} \frac{p \cdot n}{(p \cdot n)^{2}+\varepsilon^{2}} \text {. }
$$

We will use this prescription even in the massive case. We stress that if we split the propagator in two pieces as

$$
D_{\Lambda, \mu \nu}=\bar{D}_{\Lambda, \mu \nu}+\tilde{D}_{\Lambda, \mu \nu}
$$

where $\tilde{D}_{\Lambda, \mu \nu}$ is the term proportional to $n_{\mu} n_{\nu} / n^{2}$,

$$
\tilde{D}_{\Lambda, \mu \nu}(p)=-\frac{\Lambda^{2} n_{\mu} n_{\nu}}{[p \cdot n]^{2}\left(p^{2}-\Lambda^{2}+i \varepsilon\right)},
$$

we can see that our formulation reduces to the usual planar gauge expression in the $\Lambda \rightarrow 0$ limit if the $\tilde{D}_{\Lambda, \mu \nu}$ term can be neglected. This is obvious in the computation of infrared finite Feynman diagrams, far from being obvious in the computation of infrared divergent Feynman diagrams, and definitely nontrivial for physical quantities sensitive to the infrared, such as the interquark potential.

\subsection{Light-cone gauge}

The light-cone gauge is the most used and the most tested algebraic noncovariant gauge. At the present it passed many serious consistency checks and should be considered at the same level of safety of the covariant gauge, at least for what concerns the perturbative expansion in four dimensional

\footnotetext{
6 Actually in higher order computations the CPV prescription could be problematic; see the discussion in section 5.1.
} 
gauge theories. In particular it has been explicitly proved at order $O\left(g^{4}\right)$ in perturbation theory that the Wilson loop computed in the light-cone gauge gives the same result of the Wilson loop in covariant gauge, provided that we use the Mandelstam-Leibbrandt (ML) prescription to regularize the spurious singularities 19.

The Euclidean massive light-cone propagator can be formally extracted from espression (23) for light-like vectors $L_{\mu}=n_{E, \mu}$ such as $n_{E}^{2}=0$ :

$$
D_{E, \mu \nu}\left(p_{E}\right)=\frac{1}{p_{E}^{2}+\Lambda^{2}}\left\{\delta_{\mu \nu}-\frac{n_{E, \mu} p_{E, \nu}+n_{E, \nu} p_{E, \mu}}{p_{E} \cdot n_{E}}-\frac{\Lambda^{2} n_{E, \mu} n_{E, \nu}}{\left(p_{E} \cdot n_{E}\right)^{2}}\right\} .
$$

The light-cone condition can be realized in Euclidean space if the gauge vector $n_{E}=\left(n_{1}, n_{2}, n_{3}, n_{4}\right)$ has the form $n_{E}=(0,0,1, i)$ or $n_{E}^{*}=(0,0,1,-i)$, or an equivalent one after a $S O(4)$ rotation. Writing

$$
p_{3}=p_{\|} \cos \theta_{\|}, \quad p_{4}=p_{\|} \sin \theta_{\|}, \quad p_{\|}^{2}=p_{E} \cdot n_{E} p_{E} \cdot n_{E}^{*}=p_{3}^{2}+p_{4}^{2},
$$

we obtain the explicit expressions

$$
\frac{1}{p_{E} \cdot n_{E}}=\frac{1}{i p_{4}+p_{3}}=\frac{-i p_{4}+p_{3}}{p_{4}^{2}+p_{3}^{2}}=\frac{p_{E} \cdot n_{E}^{*}}{p_{\|}^{2}}=\frac{e^{-i \theta_{\|}}}{p_{\|}} .
$$

After Wick rotation in Minkowsky space $p_{0}=-i p_{4}$ we see that this approach corresponds to regularize the spurious poles with the ML prescription. The Minkowskian propagator reads

$$
-D_{\Lambda, \mu \nu}(p)=\frac{1}{p^{2}-\Lambda^{2}+i \varepsilon}\left\{g_{\mu \nu}-\frac{n_{\mu} p_{\nu}+n_{\nu} p_{\mu}}{[[p \cdot n]]}+\frac{\Lambda^{2} n_{\mu} n_{\nu}}{[[p \cdot n]]^{2}}\right\}
$$

with

$$
\frac{1}{[[p \cdot n]]} \equiv \frac{p \cdot n^{*}}{(p \cdot n)\left(p \cdot n^{*}\right)+i \varepsilon}, \quad n=(1,0,0,1), \quad n^{*}=(-1,0,0,1)
$$

and reduces to the standard one at $\Lambda \rightarrow 0$ if the $\tilde{D}_{\Lambda, \mu \nu}$ term can be neglected. Differently from the planar gauge, the light-cone choice is extremely convenient since not only it avoids the double pole problem but still mantains the advantages of the axial gauge. i.e. the transversality property which garantees the full decoupling of ghost fields,

$$
n^{\mu} D_{\mu \nu, \Lambda}(q)=0
$$


Furthermore the simple Ward identities

$$
q^{\mu} D_{\Lambda, \mu \nu}(q)=\frac{n_{\nu}}{[[n \cdot q]]}
$$

and

$$
p^{\mu} D_{\Lambda}^{\lambda \nu}(q) V_{\nu \mu \rho}(q, p,-q-p) D_{\Lambda}^{\rho \tau}(q+p)=D_{\Lambda}^{\lambda \tau}(q)-D_{\Lambda}^{\lambda \tau}(q+p)
$$

hold, where

$$
V_{\mu \nu \rho}(p, q, r)=(q-r)_{\mu} g_{\nu \rho}+(p-q)_{\rho} g_{\mu \nu}+(r-p)_{\nu} g_{\rho \mu}
$$

is the non-Abelian three-gluon vertex. By using these ingredients we explicitly checked the transversality of the gluon propagator and in general the Ward identities on the proper vertices with direct diagrammatic considerations, for any value of $\Lambda$. We repeat that in planar gauge, on the contrary, the Ward identities are much more complicated and the gluon propagator is not transverse with respect to $n_{\mu}$ or $p_{\mu}$.

It is important to stress that: i) it is impossible to impose condition (39) in covariant gauges: this is the reason why the gauge symmetry is unavoidably more complicate in the covariant case and ghost fields have to be taken into account ii) differently from planar gauge, we checked that there are no other possibile forms of the light-cone propagator consistent with (37),(38) and (39): the transversality constraint plus the symmetry requirement fixes univocally the form (35) for the light-cone propagator. In other words, equation (35) is the unique way of introducing a Wilsonian infrared cutoff in a non-Abelian gauge theory consistently with Ward identities.

\section{Problems of the pure axial gauge}

As we recalled in the introduction, the standard approach to axial gauge with the CPV prescription has various problems and in particular the test of exponentiation for a properly chosen Wilson loop fails. There has been a lot of work in the literature to solve this problem (see for instance the citation list in [15]), but in spite of this effort in our opinion at present there are no completely satisfactory solutions. For instance there is an apparently simple solution consisting in changing the prescriptions on the gluon propagator and the ghost interaction in such a way that the Wilson loop becomes the same 
as computed in covariant gauges. This is the logic of Cheng and Tsai 26] and also of the approches based on interpolating gauges, in which one try to define the axial gauge as a deformation of a more regular gauge. This kind of approaches has a long hystory (starting from the old work of [13 until very recent papers [27, 28]) nevertheless they are not completely satisfactory since i) the transversality property $n^{\mu} D_{\mu \nu}=0$ is lost; ii) the ghost fields are no longer decoupled; iii) the Ward identities have no more a simple form; iv) a careful study of the infrared singularities appearing in the limit in which the modified gauge tends to the pure axial gauge is needed. For such reasons these modifications are so drastic that in fact they should be interpreted as a switch to a truly different gauge [15]. The situation in the Wilsonian approach is different but still there are problems which will be discussed in the following sections. Our final conclusion will be that the axial gauge is definitively sick, at least in perturbation theory, and alternative gauge choices should be considered.

\subsection{The CPV regularization of the double pole}

In the Cauchy Principal Value prescription, the double pole is defined as the derivative of the single pole,

$$
\frac{1}{\left[p_{3}^{2}\right]}=-\frac{\partial}{\partial p_{3}} \frac{1}{\left[p_{3}\right]}
$$

where $1 /\left[p_{3}\right]$ is defined as in (29). This means that the double pole is regularized as

$$
\frac{1}{\left[p_{3}^{2}\right]}=\lim _{\Lambda \rightarrow 0} \frac{p_{3}^{2}-\Lambda^{2}}{\left(p_{3}^{2}+\Lambda^{2}\right)^{2}}
$$

We stress two things:

1. One could expect some problem with this regularization of the double pole, since we loose the property of the Euclidean propagator of being positive definite. This can be seen by looking at the eigenvalues of the Euclidean pure axial gauge propagator (at zero mass and with the CPV prescription), which are

$$
0, \frac{1}{p_{E}^{2}}, \frac{1}{p_{E}^{2}}, \frac{1}{\left[p_{3}\right]^{2}} .
$$


The first three eigenvalues are obviously non negative; the problem is with the last one, which is not positive definite. This means that the integral

$$
I_{3}(f)=\int_{p_{3}} \frac{1}{\left[p_{3}^{2}\right]} f\left(p_{3}\right)<0
$$

can be negative even if $f\left(p_{3}\right)$ is a regular positive function. For instance it is trivial to check that this happens for $f\left(p_{3}\right)=1 /\left(p_{3}^{2}+m^{2}\right)$. Therefore $D_{E, \mu \nu}\left(p_{E}\right)$ is no more positive definite and clearly this is a potential source of problems for the perturbative expansion.

2. The Wilsonian prescription of the double pole

$$
\frac{1}{\left[p_{3}^{2}\right]_{W}}=\lim _{\Lambda \rightarrow 0} \frac{1}{p_{3}^{2}+\Lambda^{2}}
$$

is different from the CPV prescription (42). As a matter of fact the eigenvectors of the Euclidean propagator are explicitly non-negative

$$
0, \frac{1}{p_{E}^{2}+\Lambda^{2}}, \frac{1}{p_{E}^{2}+\Lambda^{2}}, \frac{1}{p_{3}^{2}+\Lambda^{2}}
$$

and therefore one could argue that the Wilsonian prescription is better then the CPV. Unfortunately, the Wilsonian prescription has other problems that we will describe in the next section.

\subsection{The double pole problem in the Wilsonian formu- lation}

We found two major infrared problems in the Wilsonian formulation of the axial gauge: i) the Fourier transform of the propagator is divergent at $\Lambda \rightarrow 0$; ii) one loop Feynman diagrams which are infrared finite in covariant gauges becomes divergent in axial gauge for all configurations of momenta. In principle, this is not enough to prove the inconsistency of this gauge, since we should prove that at least one physical quantity is ill defined in the $\Lambda \rightarrow 0$ limit. Indeed one could think that due to miracolous cancellations related to the Ward-identities (which are respected) physical quantities are indeed finite in the $\Lambda \rightarrow 0$ limit as it happens for instance for the Wilson loop at 
order $O\left(g^{2}\right)$ (see section 7). However this is not garanteed in general. Moreover, even if this were true, the singularity of the $\Lambda \rightarrow 0$ limit would forbid in practice any numerical application, since even extremely small numerical breaking of the Ward-identities would result in enormous (at the limit infinite) differences in the final result at $\Lambda \rightarrow 0$. This is a serious drawback of the axial gauge Wilsonian formulation. We stress that, even if not noticed in those references, the problems we discuss here also apply to the formulations in [16, 17] where they are probably even worse due to the wild breaking of gauge-symmetry. They were not recognized before simply because they do not affect the computation of the one-loop beta function, which is an universal quantity not affected by the way the infrared cutoff in inserted [9] and insensitive to the gauge fixing choice.

The origin of all problems comes from the part of the propagator proportional to $p_{\mu} p_{\nu}$

$$
D_{\Lambda, \mu \nu}^{p p}=\frac{p_{\mu} p_{\nu} n^{2}}{\left(p^{2}+\Lambda^{2}\right)\left((p \cdot n)^{2}+n^{2} \Lambda^{2}\right)} .
$$

This quantity is a messy source of infrared divergencies due to the identity (in the sense of distributions)

$$
\frac{1}{(p \cdot n)^{2}+\Lambda^{2}} \stackrel{\Lambda \rightarrow 0}{=} \frac{\pi}{\Lambda} \delta(p \cdot n)
$$

This means that for any regular function $f\left(p_{3}, \Lambda\right)$ such as $f(0,0) \neq 0$ we have

$$
\lim _{\Lambda \rightarrow 0} \int_{p_{3}} \frac{f\left(p_{3}, \Lambda\right)}{p_{3}^{2}+\Lambda^{2}}=\lim _{\Lambda \rightarrow 0} \frac{f(0,0)}{2 \Lambda}=\infty .
$$

We will see in next subsections the disastrous consequences of this fact on the computation of various quantities.

\subsection{The $x$-space propagator.}

Here we show that even if the $x$-space propagator

$$
D_{\Lambda, \mu \nu}(x)=\int_{p} e^{-i p \cdot x} D_{\Lambda, \mu \nu}(p)
$$


is perfectly defined for any $\Lambda \neq 0$, the infrared limit $\Lambda \rightarrow 0$ does not exist due to a strong infrared divergence coming from the double pole part of the propagator proportional to $p_{\mu} p_{\nu}$ (the double pole part proportional to $\Lambda^{2} n_{\mu} n_{\nu}$ and the single pole part are regular as $\Lambda \rightarrow 0$ ). This can be proved with a direct computation by using the result (49). One obtains $D_{\Lambda, i 3}=D_{\Lambda, 3 i}=$ $D_{\Lambda, 33}=0$ and

$$
D_{\Lambda, i j}(x) \stackrel{\Lambda \rightarrow 0}{=} \frac{1}{2 \Lambda} \int_{\bar{p}} \frac{p_{i} p_{j}}{\bar{p}^{2}} e^{-i \bar{p} \cdot \bar{x}}=\frac{1}{2 \Lambda} \frac{1}{3} \delta_{i j} \delta^{(3)}(\bar{x}) \stackrel{\Lambda \rightarrow 0}{=} \infty
$$

Therefore the Fourier transform of the propagator does not exist at $\Lambda \rightarrow 0$.

The level of danger of this problem is unclear at this level, since it could not affect physical quantities. We will see in section 7 that the simplest physical quantity we can compute, i.e. the Wilson loop at order $O\left(g^{2}\right)$, only depends on the Fourier transform of the transverse part of the propagator,

$$
D_{\Lambda, \mu \nu}^{T}(x) \equiv \int_{p} e^{-i p \cdot x} t_{\mu \lambda}(p) D_{\Lambda}^{\lambda \rho}(p) t_{\rho \nu}(p),
$$

which is safe in the limit $\Lambda \rightarrow 0$. To show this point we have to prove that the $\tilde{D}_{\mu \nu, \Lambda}(x)$ part of the propagator,

$$
\tilde{D}_{\Lambda, \mu \nu}(x)=D_{\Lambda}^{n n}(x) \frac{n_{\mu} n_{\nu}}{n^{2}}
$$

which is the only one which transverse part depends on the gauge vector $n_{\mu}$ is vanishing at $\Lambda \rightarrow 0$. A direct computation in Euclidean space gives

$$
D_{\Lambda}^{n n}\left(x_{E}\right)=\int_{p_{E}} \frac{\exp \left(i p_{E} \cdot x_{E}\right)}{p_{E}^{2}+\Lambda^{2}}\left[-\frac{\Lambda^{2}}{p_{3}^{2}+\Lambda^{2}}\right] .
$$

By direct inspection using (48) one sees that this term is linearly vanishing with $\Lambda$; therefore the infrared limit of the transverse part of the propagator exists finite and is independent of the gauge vector $n_{\mu}$ :

$$
D_{\Lambda, \mu \nu}^{T}\left(x_{E}\right) \stackrel{\Lambda \rightarrow 0}{=}\left(\delta_{\mu \nu}-\frac{\partial_{E, \mu} \partial_{E, \nu}}{\partial_{E}^{2}}\right) \frac{1}{4 \pi^{2} x_{E}^{2}} .
$$

The crucial point here is the fact that the singular $D_{\Lambda, \mu \nu}^{p p}$ term (47) does not contributes to the transverse part of the propagator. 


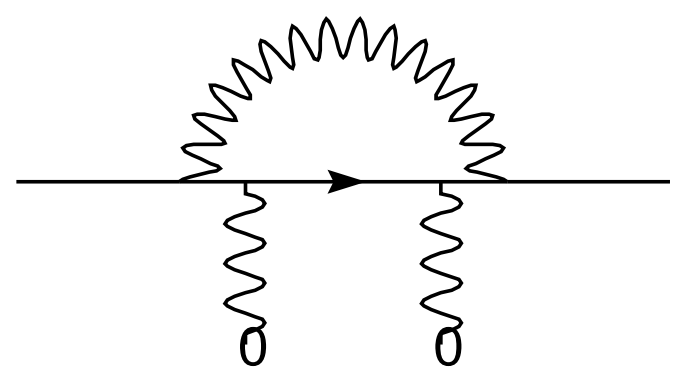

Figure 1: Self-energy with two zero-momentum insertions.

\subsection{Computation of a one-loop integral}

The simplest perturbative quantity sensitive to the double pole problem is the one-loop quark self-energy. In this section we will see that the $p_{\mu} p_{\nu}$ part of the propagator gives an infinite contribution to this quantity in the $\Lambda \rightarrow 0$ limit. Unfortunately this is a gauge-dependent quantity therefore in principle we cannot positively conclude about the inconsistency of the axial gauge choice with the Wilsonian prescription (45). However in practice this is a serious drawback since it forbids the direct computation of any perturbative quantity except the one-loop anomalous dimensions and the beta function [9].

In order to simplify the computation, by avoiding the inessential complications with the gamma matrices, we consider the self-energy of a scalar quark. Moreover, in order to avoid infrared divergences we take a quark mass $m \neq 0$ and in order to avoid ultraviolet divergences we derive twice with respect to $m^{2}$. In this way we obtain a quantity corresponding to the four-point vertex with two insertions at zero momentum shown in figure 1. This quantity, which we denote by

$$
F\left(\bar{p}^{2}, p_{3}^{2}, \Lambda\right)=\int_{q_{E}}\left(\frac{\partial}{\partial m^{2}}\right)^{2}\left[\frac{\left(2 p_{E}+q_{E}\right)^{\mu}\left(2 p_{E}+q_{E}\right)^{\nu}}{\left[\left(q_{E}+p_{E}\right)^{2}+m^{2}+\Lambda^{2}\right]} D_{\mu \nu}\left(q_{E}, \Lambda\right)\right]
$$

is ultraviolet convergent and (in covariant gauges) infrared finite even at $\Lambda=0$ if $p_{E}^{2} \neq-m^{2}$. Still, the $\Lambda \rightarrow 0$ limit is singular in the massive axial gauge since the dominant contribution comes from the double pole part of 
the propagator which, due to equation (49), is infinity in the $\Lambda \rightarrow 0$ limit:

$$
F\left(\bar{p}^{2}, p_{3}^{2}, \Lambda\right) \stackrel{\Lambda \rightarrow 0}{=}-\frac{1}{2 \Lambda} \int_{\vec{q}} \frac{2}{\left[(\bar{q}+\bar{p})^{2}+m^{2}\right]^{3}} q_{i} q_{j}(2 p+q)^{i}(2 p+q)^{j} \rightarrow \infty .
$$

Notice that this problem appears even in the Abelian theory. Moreover it is clear that the problem becomes worse in the non-Abelian case and at higher orders in perturbation theory. Still, in principle it is possible that cancellations allows to define unambigously physical quantities even in the $\Lambda \rightarrow 0$ limit, since the various Feynman diagrams should add in such a way that the final result at $\Lambda \rightarrow 0$ is finite and independent of the gauge fixing, i.e. the same that in covariant gauges. However, this is an highly non-trivial point and cannot be taken for granted at finite order in perturbation theory.

\section{The planar gauge}

For the reasons described above, we think that it is not convenient to insist on the axial gauge and from now on we switch to other gauge choices. The simplest examples where the difficulties of the axial gauge can be circumvented (at least in the standard massless formalism and at order $O\left(g^{4}\right)$ in perturbation theory) are the planar gauge and the light-cone gauge. We consider first the planar gauge.

\subsection{Remarks on the planar gauge}

Properly speaking, even the standard massless planar gauge choice with the CPV prescription is not completely controled and the consistency of the perturbative expansion in this gauge is an open problem; nevertheless it will be studied in this section for sake of comparison with the axial gauge and the light-cone gauge choices.

The reason of the difficulties can be traced back to the well known splitting formula [15]

$$
\frac{1}{[n k]} \frac{1}{[n(p-k)]}=\frac{1}{n p}\left(\frac{1}{[n k]}+\frac{1}{[n(p-k)]}\right)-\pi^{2} \delta(n k) \delta(n p) .
$$

Due to the presence of the delta function terms, in two-loops or higher orders 
computations involving gluon vertices there appear ill-defined objects of kind

$$
\frac{1}{[k n]} \delta(k n) \sim \frac{k n}{(k n)^{2}+\varepsilon^{2}} \frac{\varepsilon / \pi}{(k n)^{2}+\varepsilon^{2}}
$$

which have to be studied in detail. It is known that in the massless computation of the $O\left(g^{4}\right)$ Wilson loop there is a precise way to manage these terms and the final result is consistent with the covariant gauge [18. Nevertheless, the situation at order $O\left(g^{6}\right)$ is unknown and in general the definition of two-loops Feynman diagrams is problematic. This is the reason why we will switch to the light-cone gauge in the next section. However it is interesting to see that even at the level of the planar gauge the divergences found before in one-loop computations are absent, essentially because the double pole term proportional to $p_{\mu} p_{\nu}$ in (47) is absent. There is however a double pole in the $\tilde{D}_{\Lambda, \mu \nu}$ part of the propagator, but since this term is multiplied by a $\Lambda^{2}$ factor it is espected to vanish in the $\Lambda \rightarrow 0$ limit. This is indeed the case for infrared safe quantities and it will be explicitly verified in the examples we consider here. We stress that this is by no means a trivial statement, since in the computation of particular infrared divergent quantities the $\tilde{D}_{\Lambda, \mu \nu}$ term in the denominator could be not subleading with respect to the other terms and actually could also give the dominant contribution. We will see in section 6 that this is indeed the case in the light-cone gauge. Moreover we will see in section 7 that the $\tilde{D}_{\Lambda, \mu \nu}$ term contributes to the Wilson loop and is suppressed only if the $\Lambda \rightarrow 0$ limit is done properly, i.e. at finite $T$.

\subsection{The $x$-space propagator}

We prove here that the Fourier transform of the propagator is well defined and in the $\Lambda \rightarrow 0$ limit reduces to the Fourier transform of the standard planar gauge propagator. This is obvious for the $\bar{D}_{\Lambda, \mu \nu}$ part of the propagator, therefore we have simply to prove that the Fourier transform of the $\tilde{D}_{\Lambda, \mu \nu}$ term is vanishing at $\Lambda \rightarrow 0$. A direct computation gives

$$
\lim _{\Lambda \rightarrow 0} \Lambda^{2} \int_{\bar{p}, p_{3}} \frac{\exp \left(i p_{3} x_{3}+i \bar{p} \cdot \bar{x}\right)}{\left[p_{3}\right]^{2}\left(p_{3}^{2}+\bar{p}^{2}+\Lambda^{2}\right)}=\lim _{\Lambda \rightarrow 0} \frac{\Lambda^{2}}{8 \pi^{2}} \ln \left(\Lambda^{2} \bar{x}^{2}\right)=0
$$

This result can be obtained by first doing the $p_{3}$ integral using $1 /\left[p_{3}^{2}\right]=$ $\left[p_{3}\right]^{-1} \partial_{p_{3}}$; the successive three-dimensional integral generates an infrared logarithimic singularity at $\Lambda \rightarrow 0$ which is killed by the $\Lambda^{2}$ prefactor. Therefore 
we have solved the problem with the Fourier transform. However we stress again that in the computation of infrared sensitive quantities this term in general could gives an essential contribution to the final result. Furthermore, we will see in section 7 that this term is suppressed in the Wilson loop computation only if the $\Lambda \rightarrow 0$ and $T \rightarrow \infty$ limit are done in the correct order. Therefore the relation between the massive version of the planar gauge in the $\Lambda \rightarrow 0$ limit and the standard planar gauge in general is delicate.

\subsection{Computation of a one-loop finite quantity}

Now we can study what happens in the computation of the quark self-energy with two zero-momentum insertions, i.e. the analogous of the quantity $F$ defined in (56). If we split the Euclidean planar gauge propagator in three terms (in this section for notational simplicity we write $p$ for $p_{E}$ )

$$
\begin{aligned}
D_{\mu \nu}(p, \Lambda) & =\frac{1}{p^{2}+\Lambda^{2}}\left\{\delta_{\mu \nu}-\frac{n_{\mu} p_{\nu}+n_{\nu} p_{\mu}}{[p \cdot n]}-\frac{\Lambda^{2} n_{\mu} n_{\nu}}{[p \cdot n]^{2}}\right\} \\
& =D_{\mu \nu}^{(a)}(p, \Lambda)+D_{\mu \nu}^{(b)}(p, \Lambda)+D_{\mu \nu}^{(c)}(p, \Lambda)
\end{aligned}
$$

we can split the computation in three different contributions:

$$
F\left(\bar{p}^{2}, p_{3}^{2}, \Lambda\right)=F^{(a)}\left(p^{2}, \Lambda\right)+F^{(b)}\left(\bar{p}^{2}, p_{3}^{2}, \Lambda\right)+F^{(c)}\left(\bar{p}^{2}, p_{3}^{2}, \Lambda\right)
$$

with

$$
\begin{gathered}
F^{(a)}\left(p^{2}, \Lambda\right)=\int_{q}\left(\frac{\partial}{\partial m^{2}}\right)^{2} \frac{(2 p+q)^{2}}{\left(q^{2}+\Lambda^{2}\right)\left((q+p)^{2}+m^{2}+\Lambda^{2}\right)} \\
F^{(b)}\left(\bar{p}^{2}, p_{3}^{2}, \Lambda\right)=\int_{q}\left(\frac{\partial}{\partial m^{2}}\right)^{2} \frac{-2(2 p+q) \cdot n(2 p+q) \cdot q}{[q \cdot n]\left(q^{2}+\Lambda^{2}\right)\left((q+p)^{2}+m^{2}+\Lambda^{2}\right)} \\
F^{(c)}\left(\bar{p}^{2}, p_{3}^{2}, \Lambda\right)=\int_{q}\left(\frac{\partial}{\partial m^{2}}\right)^{2} \frac{-\Lambda^{2}(2 p \cdot n+q \cdot n)^{2}}{[q \cdot n]^{2}\left(q^{2}+\Lambda^{2}\right)\left((q+p)^{2}+m^{2}+\Lambda^{2}\right)} .
\end{gathered}
$$

The first contribution is the same as in Feynman gauge and can be computed using the standard Feynman parametrization. It gives

$$
F^{(a)}\left(p^{2}, \Lambda\right)=\frac{1}{16 \pi^{2}} \int_{0}^{1} d x(1-x)^{2} \frac{p^{2}\left(1+4 x-x^{2}\right)+2\left[m^{2}(1-x)+\Lambda^{2}\right]}{\left[\left(p^{2} x+m^{2}\right)(1-x)+\Lambda^{2}\right]^{2}}
$$


where the $x$-integral can be performed in terms of elementary functions (logarithms). In particular in the on-shell limit $p^{2} \rightarrow-m^{2}, \Lambda \rightarrow 0$ the $x \simeq 1$ integration region dominates and we have

$$
F^{(a)}\left(p^{2}, \Lambda=0\right)=-\frac{1}{4 \pi^{2}} \frac{1}{p^{2}+m^{2}}+O\left(\left(p^{2}+m^{2}\right)^{0}\right)
$$

if the $\Lambda \rightarrow 0$ limit is taken first. On the contrary, if the $p^{2} \rightarrow-m^{2}$ limit is taken first, we have

$$
F^{(a)}\left(p^{2}=-m^{2}, \Lambda\right)=-\frac{1}{16 \pi} \frac{1}{m \Lambda}+O\left(\frac{\Lambda^{0}}{m^{2}}\right) .
$$

This is an explicit confirmation of the fact the the order of limits is a delicate question for infrared divergent quantities.

The second contribution is typical of planar gauge. Its general expression is rather complicate, but there is a simplification if we restrict the analysis to the limits $\left|\bar{p}^{2}\right| \gg p_{3}^{2}$ or $\left|\bar{p}^{2}\right| \ll p_{3}^{2}$. The first limit $\left|\bar{p}^{2}\right| \gg p_{3}^{2}$ is trivial in the sense that the integral can be recast in a covariant-like form and its computation is no more difficult than the computation of the covariant term and gives a completely analogous contribution. In the second limit $\left|\bar{p}^{2}\right| \ll p_{3}^{2}$ instead the peculiarities of the planar gauge are evident. In particular the quantity

$$
F^{(b)}\left(p_{3}^{2}, \Lambda\right)=\lim _{\bar{p} \rightarrow 0} F^{(b)}\left(\bar{p}^{2}, p_{3}^{2}\right)
$$

can be computed with the method of double Feynman parametrization discussed in appendix B. After some manipulation it reads

$$
F^{(b)}\left(p_{3}^{2}, \Lambda\right)=\int_{q_{3}, \tilde{q}} \int_{0}^{1} d x \int_{1-x}^{1} d z\left(\frac{\partial}{\partial m^{2}}\right)^{2} \frac{N\left(q_{\|}^{2}, \tilde{q}^{2}, p_{3}^{2}, x, z\right)}{D\left(q_{\|}^{2}, \tilde{q}^{2}, p_{3}^{2}, m^{2}, \Lambda^{2}, x, z\right)}
$$

with

$$
N=-2\left[p_{3}^{4}\left(1-x^{2}\right)^{2}+p_{3}^{2}\left[2\left(3 x^{2}-1\right) q_{3}^{2}-\tilde{q}^{2}\left(1-x^{2}\right) / z\right]+q_{3}^{2}\left[q_{3}^{2}+\tilde{q}^{2} / z\right]\right]
$$

and

$$
D=z^{3 / 2}\left[q_{3}^{2}+\tilde{q}^{2}+\Lambda^{2} z+\left(p_{3}^{2} x+m^{2}\right)(1-x)\right]^{3} .
$$


Now the integrations in $q_{3}, \tilde{q}$ and $z$ are straightforward (see appendix B) and all the complexity is confined into the $x$-integration. In the $\Lambda \rightarrow 0$ limit there is a strong simplification and it can be exactly performed in terms of elementary functions. The final result is

$$
\begin{aligned}
F^{(b)}\left(p_{3}^{2}, \Lambda=0\right) & =-\frac{1}{4 \pi^{2} p_{3}^{2}}-\frac{p_{3}^{2} / m^{2}-1}{8 \pi^{2}\left(p_{3}^{2}+m^{2}\right)} \\
& -\frac{\sqrt{p_{3}^{2}} \ln \left[\left(\sqrt{p_{3}^{2}+m^{2}}-\sqrt{p_{3}^{2}}\right) /\left(\sqrt{p_{3}^{2}}+\sqrt{p_{3}^{2}+m^{2}}\right)\right]}{8 \pi^{2}\left(p_{3}^{2}+m^{2}\right)^{3 / 2}} .
\end{aligned}
$$

The third contribution is characteristic of the Wilsonian formulation and must vanish in the $\Lambda \rightarrow 0$ limit in order to recover the results of the standard massless planar gauge:

$$
\lim _{\Lambda \rightarrow 0} F^{(c)}\left(p_{E}\right)=0 .
$$

This is immediate to see in the limit $\left|\bar{p}^{2}\right| \ll p_{3}^{2}$. In the opposite limit $\left|\bar{p}^{2}\right| \gg p_{3}^{2}$ one could expect divergences at $\bar{p}^{2}=-m^{2}$; but it is immediate to see that

$$
F^{(c)}\left(\bar{p}^{2}=-m^{2}, \Lambda\right) \sim \frac{\Lambda^{2}}{m^{2}} F^{(a)}\left(\bar{p}^{2}=-m^{2}, \Lambda\right)
$$

therefore $F^{(c)}\left(\bar{p}^{2}=-m^{2}, \Lambda\right)$ is linearly vanishing at $\Lambda \rightarrow 0$. Thus the $\tilde{D}_{\Lambda, \mu \nu}$ term can always be neglected, at least in this kind of one-loop computations. We will see that this is not always the case for the light-cone gauge.

\section{Safeness of the light-cone gauge}

For the point of view of the consistency of the perturbative expansion the light-cone gauge is expected to be the safest choice. There are various reasons for this expectation, which we will discuss now.

The first reason is the fact that, contrary to the planar gauge, the Euclidean propagator is (semi) positive definite. This can be immediately proved by solving the eigenvalue equation

$$
\operatorname{det}\left(\lambda \delta_{\nu}^{\mu}-D_{\nu}^{\mu}\left(p_{E}, n_{E} ; \Lambda\right)\right)=0
$$

which after explicit computation reads

$$
\lambda^{2}\left(\lambda-\frac{1}{p_{E}^{2}+\Lambda^{2}}\right)^{2}=0
$$


with solutions

$$
\lambda_{1}=\lambda_{2}=\frac{1}{p_{E}^{2}+\Lambda^{2}}, \quad \lambda_{3}=\lambda_{4}=0
$$

From this computation one sees that the eigenvalues $\lambda_{i}=\lambda_{i}\left(p_{E}, n_{E}\right)$ are nonnegative. In addition, they depend only on the Lorentz-invariant combination $p_{E}^{2}$ and not on $n_{E}$. We emphasize that this is not true in non-covariant gauges others than the light-cone one. Therefore one expects a somehow less prononced Lorentz breaking in the light-cone gauge.

The second reason is that within the ML prescription the splitting formula (58) holds without the delta function term: as a consequence there are no ill defined terms in higher orders of perturbation theory as it happens with the CPV prescription in axial and planar gauges.

The third reason is that the massless limit of the light-cone gauge is expected to be less singular than in others gauges. This expectation comes from the that the Fourier transform of the $\tilde{D}_{\Lambda, \mu \nu}$ term is quadratically vanishing as $\Lambda^{2} \rightarrow 0$ : this should be contrasted with the axial gauge case where the suppression of the $\tilde{D}_{\Lambda, \mu \nu}$ term in only linear in $\Lambda$ (see the discussion after Eq. (54)) and the planar gauge where this term vanishes as $\Lambda^{2}$ times a logarithm. We will discuss in detail this latter point just below.

\subsection{The $x$-space propagator}

In order to explicitly compute the Fourier transform of the $\tilde{D}_{\Lambda, \mu \nu}(x)$ term we have to study the Euclidean integral

$$
D_{\Lambda}^{n n}\left(x_{E}\right)=-\Lambda^{2} \int_{q_{E}} \frac{e^{i q_{E} \cdot x_{E}}}{\left(q_{E} \cdot n_{E}\right)^{2}\left(q_{E}^{2}+\Lambda^{2}\right)} .
$$

We proceed as follow. First, we introduce the angles $\theta$ and $\theta^{\prime}$ such as

$$
q_{1}=q_{\perp} \cos \theta^{\prime}, \quad q_{2}=q_{\perp} \sin \theta^{\prime}, \quad q_{3}=q_{\|} \sin \theta, \quad q_{4}=q_{\|} \cos \theta
$$

and we take $x=\left(x_{\perp}, 0, x_{\|}, 0\right)$ (this is not restrictive); then the Fourier transform can be written

$$
D_{\Lambda}^{n n}\left(x_{E}\right)=-\Lambda^{2} \int \frac{q_{\perp} d q_{\perp} d \theta^{\prime}}{(2 \pi)^{2}} \frac{d q_{\|}}{(2 \pi)^{2}} \frac{e^{-2 i \theta-i q_{\|} x_{\|} \sin \theta-i q_{\perp} x_{\perp} \sin \theta^{\prime}}}{q_{\|}\left(q_{\|}^{2}+q_{\perp}^{2}+\Lambda^{2}\right)} .
$$


$D_{\Lambda}^{n n}\left(x_{E}\right)$ can be computed by first performing the angular integrations and then the momentum integrations in $q_{\|}$and $q_{\perp}$. The angular integrations can be done by using the following representation of Bessel functions

$$
J_{n}(z)=\frac{1}{2 \pi} \int_{0}^{2 \pi} d \theta e^{-i n \theta-i z \sin \theta}
$$

for $n=0$ and $n=2$ respectively. In this way we obtain

$$
D_{\Lambda}^{n n}\left(x_{E}\right)=-\frac{\Lambda^{2}}{(2 \pi)^{2}} \int_{0}^{\infty} d q_{\perp} q_{\perp} \int_{0}^{\infty} \frac{d q_{\|}}{q_{\|}} \frac{J_{2}\left(q_{\|} x_{\|}\right) J_{0}\left(q_{\perp} x_{\perp}\right)}{q_{\|}^{2}+q_{\perp}^{2}+\Lambda^{2}} .
$$

Since at small $z$ the Bessel function $J_{2}(z) \simeq \frac{1}{8} z^{2}$ is quadratically vanishing whereas at large $z$ is exponentially suppressed, we see that the $q_{\|}$-integral is infrared and ultraviolet finite. It can be exactly computed with the result

$$
D_{\Lambda}^{n n}\left(x_{E}\right)=-\frac{\Lambda^{2}}{8 \pi^{2}} \int_{0}^{\infty} d q_{\perp} q_{\perp} \frac{\omega_{\perp}^{2}-4 x_{\|}^{-2}+2 \omega_{\perp}^{2} K_{2}\left(\omega_{\perp} x_{\|}\right)}{\omega_{\perp}^{4}} J_{0}\left(q_{\perp} x_{\perp}\right),
$$

with $\omega_{\perp}^{2} \equiv q_{\perp}^{2}+\Lambda^{2}$. Using the series expansion of the Bessel functions

$$
K_{2}(z)=\frac{4-z^{2}}{2 z^{2}}+O\left(z^{2} \log z^{2}\right), \quad J_{0}(z)=1-\frac{1}{4} z^{2}+O\left(z^{4}\right),
$$

one sees that the $q_{\perp}$-integral is also finite, even in the $\Lambda \rightarrow 0$ limit where it can be computed exactly and it gives

$$
D_{\Lambda}^{n n}\left(x_{E}\right)=-\frac{\Lambda^{2}}{16 \pi^{2}}\left[\frac{x_{E}^{2}}{x_{\|}^{2}} \log \frac{x_{E}^{2}}{x_{\perp}^{2}}-1+O\left(\Lambda^{2} x_{\perp}^{2}, \Lambda^{2} x_{\|}^{2}\right)\right]
$$

where $x_{E}^{2}=x_{\|}^{2}+x_{\perp}^{2}$. Thus, $\tilde{D}_{\Lambda, \mu \nu}(x)$ is quadratically vanishing in the $\Lambda \rightarrow 0$ limit. We also see that there could be problems in the limit $x_{\|} / x_{\perp} \rightarrow \infty$ which is relevant for the Wilson loop computation. This point will be discussed in detail in section 7 .

\subsection{The structure of (soft) infrared divergences}

It is possible to repeat the computation of section 5.3 in the light-cone gauge. The covariant contribution $F^{(a)}(p, \Lambda)$ is obviously the same, whereas the contributions $F^{(b)}(p, \Lambda)$ and $F^{(c)}(p, \Lambda)$ are different from the analogous one 
in planar gauge. Still, one can use the double Feynman parametrization method described in appendix B. In the limit $p_{\perp}^{2} / p_{\|}^{2} \rightarrow 0$ the contribution $F^{(b)}$ can be rewritten in the form

$$
F^{(b)}\left(p_{\|}^{2}, \Lambda\right)=\int_{q_{\|}, \tilde{q}_{\perp}} \int_{0}^{1} d x \int_{1-x}^{1} d z\left(\frac{\partial}{\partial m^{2}}\right)^{2} \frac{N\left(q_{\|}^{2}, \tilde{q}_{\perp}^{2}, p_{\|}^{2}, x, z\right)}{D\left(q_{\|}^{2}, \tilde{q}_{\perp}^{2}, p_{\|}^{2}, m^{2}, \Lambda^{2}, x, z\right)}
$$

with

$$
N\left(q_{\|}^{2}, \tilde{q}_{\perp}^{2}, p_{\|}^{2}, x, z\right)=-8 q_{\|}^{2} p_{\|}^{2}+\Delta N\left(q_{\|}^{2}, \tilde{q}_{\perp}^{2}, p_{\|}^{2}, x, z\right)
$$

and

$$
D=z\left[q_{\|}^{2}+\tilde{q}_{\perp}^{2}+\Lambda^{2} z+\left(p_{\|}^{2} x+m^{2}\right)(1-x)\right]^{3} .
$$

Actually, we exactly computed the expression $\Delta N\left(q_{\|}^{2}, \tilde{q}_{\perp}^{2}, p_{\|}^{2}, x, z\right)$ in the numerator and it is possible to perform exactly the integrals in the general case, but the resulting expressions are lenghty and they will not be reported here. In order to analyze the on-shell divergences at $p^{2} \simeq-m^{2}$ the only relevant term is the fist one displayed in the right hand side of (87). The integrations in $q_{\|}$and $q_{\perp}$ are straighforward and they give, after neglecting the $\Delta N$ term,

$$
F^{(b)}\left(p_{\|}^{2}, \Lambda\right) \simeq-\frac{1}{4 \pi^{2}} \int_{0}^{1} d x \int_{1-x}^{1} \frac{d z}{z} \frac{p_{\|}^{2}(1-x)^{2}}{\left[\Lambda^{2} z+\left(p_{\|}^{2} x+m^{2}\right)(1-x)\right]^{2}} .
$$

If we take the $\Lambda \rightarrow 0$ limit first we obtain

$$
F^{(b)}\left(p_{\|}^{2}, \Lambda=0\right) \stackrel{p^{2} \rightarrow-m^{2}}{=}-\frac{1}{4 \pi^{2}} \frac{\ln \left[\left(p_{\|}^{2}+m^{2}\right) / p_{\|}^{2}\right]}{p_{\|}^{2}+m^{2}}+O\left(\frac{1}{p_{\|}^{2}+m^{2}}\right)
$$

and this is dominant with respect to the divergence $1 /\left(p^{2}+m^{2}\right)$ coming from the covariant contribution $F^{(a)}$. If we take the $p^{2} \rightarrow-m^{2}$ limit first we obtain

$$
F^{(b)}\left(p_{\|}^{2}=-m^{2}, \Lambda\right)=\frac{1}{8 \pi^{2}} \frac{1}{\Lambda^{2}}+O\left(\frac{\Lambda^{0}}{m^{2}}\right)
$$

which is still dominant with respect to the linear divergence coming from the covariant contribution $F^{(a)}$. 
Now we can study in an analogous way the contribution of the $\tilde{D}_{\Lambda, \mu \nu}$ part of the propagator, i.e. the $F^{(c)}$ term. It is obvious that this term is quadratically vanishing at $\Lambda \rightarrow 0$ if $p^{2} \neq-m^{2}$. Nevertheless, if $p^{2}=-m^{2}$, this term could be divergent as $\Lambda \rightarrow 0$. This is the case indeed. Actually it is easy to understand that there is no divergence in the limit $\left|p_{\|}^{2}\right| \ll p_{\perp}^{2}$, using the same argument as in equation (75). However in the opposite limit $\left|p_{\|}^{2}\right| \gg p_{\perp}^{2}$ the infrared divergence is enhanced and actually dominates with respect to the covariant contribution. To see this point we write down the explicit expression of $F^{(c)}$ in the near on-shell region, obtained with the method of double Feynman parametrization. It reads

$$
F^{(c)}\left(p_{\|}^{2}, \Lambda\right)=\int_{q_{\|}, \tilde{q}_{\perp}} \int_{0}^{1} d x \int_{1-x}^{1} d z\left(\frac{\partial}{\partial m^{2}}\right)^{2} \frac{\tilde{N}\left(q_{\|}^{2}, \tilde{q}_{\perp}^{2}, p_{\|}^{2}, x, z\right)}{\tilde{D}\left(q_{\|}^{2}, \tilde{q}_{\perp}^{2}, p_{\|}^{2}, m^{2}, \Lambda^{2}, x, z\right)}
$$

with

$$
\tilde{N}\left(q_{\|}^{2}, \tilde{q}_{\perp}^{2}, p_{\|}^{2}, x, z\right)=24(1-x)^{2} p_{\|}^{4} \Lambda^{2}+\Delta \tilde{N}\left(q_{\|}^{2}, \tilde{q}_{\perp}^{2}, p_{\|}^{2}, x, z\right)
$$

and

$$
\tilde{D}=z\left[q_{\|}^{2}+\tilde{q}_{\perp}^{2}+\Lambda^{2} z+\left(p_{\|}^{2} x+m^{2}\right)(1-x)\right]^{4} .
$$

In the term $\Delta \tilde{N}\left(q_{\|}^{2}, \tilde{q}_{\perp}^{2}, p_{\|}^{2}, x, z\right)$ in the numerator we collected all the contributions which are subleading in the on-shell limit. After double derivation with respect to the mass $m^{2}$ and momentum integration we obtain

$$
F^{(c)}\left(p_{\|}^{2}, \Lambda \rightarrow 0\right) \stackrel{p_{\|}^{2} \rightarrow-m^{2}}{=} \frac{3}{2 \pi^{2}} \int_{0}^{1} d x \int_{1-x}^{1} \frac{d z}{z} \frac{\Lambda^{2} p_{\|}^{4}(1-x)^{4}}{\left[\Lambda^{2} z+\left(p_{\|}^{2} x+m^{2}\right)(1-x)\right]^{4}}
$$

In order to study the $\Lambda \rightarrow 0$ limit at $p_{\|}^{2}=-m^{2}$ it is convenient do rescale the $z$ variable as

$$
z=\tilde{z}(1-x), \quad \int_{1-x}^{1} \frac{d z}{z}=\int_{1}^{1 /(1-x)} \frac{d \tilde{z}}{\tilde{z}}
$$

We see that for $x \rightarrow 1$ the upper limit of the $\tilde{z}$-integral tends to infinity, therefore the $z$-integration simplifies. Then the $x$-integration is done taking 
in account that the $x \simeq 1$ integration region dominates in the near on-shell region; finally one obtains

$$
F^{(c)}\left(p_{\|}^{2}=-m^{2}, \Lambda\right) \stackrel{\Lambda \rightarrow 0}{=} \frac{1}{6 \pi^{2}} \frac{m^{2}}{\Lambda^{4}} .
$$

It is important to notice that even if the $\tilde{D}_{\Lambda}$ term dominates the soft divergence at $m \neq 0$, it can be always neglected in the analysis of hard or collinear divergences, i.e. if $m=0$. In this case in fact the contribution from the $\tilde{D}_{\Lambda}$ term is quadratically vanishing. This point can be understood with a dimensional argument and explicitly checked by using the double Feynman parametrization formula and by noticing that at $m^{2}=0$ it is the $x \simeq 0$ region of integration which dominates, not the $x \simeq 1$ region. Then the $z$-integration can be done by using

$$
\int_{1-x}^{1} \frac{d z}{z} f(x, z) \simeq x f(x, 1), \quad x \simeq 0
$$

and the $x$-integration by using the tricks reported in appendix B. With similar techniques we checked that the gluon self-energy integral in the $\Lambda \rightarrow 0$ limit goes smoothly to the standard massless integral for off-shell Euclidean momenta $p_{\|}^{2} \neq-p_{\perp}^{2}$.

\section{The Wilson loop test}

The Wilson loop is the simplest physical quantity where the effects and the problems of the infrared regularization can be studied. In particular our scope here is to study the subtilities of the $\Lambda \rightarrow 0$ limit and to test how the essential property of the gauge-invariance of the Wilson loop is recovered when the infrared cutoff is removed. In concrete in this section we compute the Wilson up to order $O\left(g^{2}\right)$ in perturbation theory. This is enough for elucidating various important features of massive axial, planar and lightcone gauges and it is a first step versus a more comprehensive computation at order $O\left(g^{4}\right)$ in perturbation theory.

For definiteness, we shall consider a rectangular Wilson loop $\Gamma_{L T}$ of size $2 L \times 2 T$, with $T \gg L$. We shall work in Euclidean space with coordinates $x_{1}, x_{2}, x_{3}, x_{4}$ and we shall take the loop in the plane $x_{2} x_{3}$, as shown in figure 2. We should notice that $T$ denotes a lenght in the spatial direction $x_{3}$, i.e. 


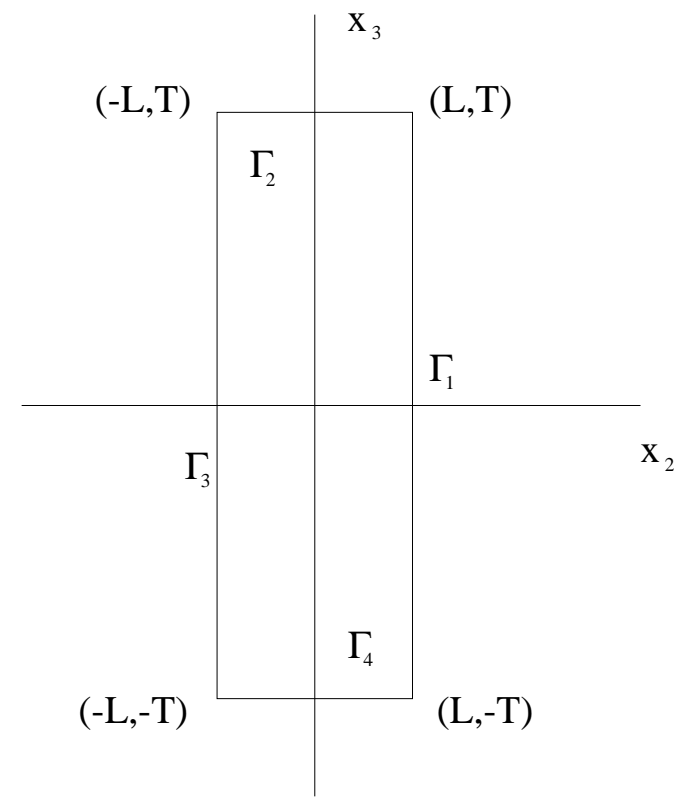

Figure 2: Rectangular Wilson loop of size $2 L \times 2 T$.

the direction of the gauge vector $n_{E}^{\mu}=(0,0,1,0)$, and not in the temporal direction $x_{4}=i x_{0}$. Therefore apparently this loop in not related to the interquark potential. Nevertheless if the theory is consistent all directions in the Euclidean space must be physically equivalent at $\Lambda \rightarrow 0$, therefore the final result must be the same that for the loop corresponding to the interquark potential where the side $T$ is in the temporal direction.

Formally the Wilson loop is defined by

$$
W_{\Gamma_{L T}}=\frac{1}{N_{c}}<\operatorname{Tr} P \exp \left(i g \int_{\Gamma_{L T}} A_{\mu} d x^{\mu}\right)>
$$

where $P$ denotes the path ordering on the loop $\Gamma_{L T}$, Tr is the trace in the fundamental representation of $S U\left(N_{c}\right)$ and the average is evaluated via a perturbative expansion of the Euclidean functional integral. In our case $\Gamma_{L T}$ can be split in four pieces

$$
\Gamma_{L T}=\Gamma^{(1)}+\Gamma^{(2)}+\Gamma^{(3)}+\Gamma^{(4)},
$$


parametrized as

$$
\begin{aligned}
& \Gamma^{(1)}(s)=s T\left(\begin{array}{c}
0 \\
0 \\
+1 \\
0
\end{array}\right), \quad \Gamma^{(2)}(s)=s L\left(\begin{array}{c}
0 \\
-1 \\
0 \\
0
\end{array}\right), \\
& \Gamma^{(3)}(s)=s T\left(\begin{array}{c}
0 \\
0 \\
-1 \\
0
\end{array}\right), \quad \Gamma^{(4)}(s)=s L\left(\begin{array}{c}
0 \\
+1 \\
0 \\
0
\end{array}\right),
\end{aligned}
$$

where the parameter $s$ lives in the interval $[-1,1]$. Equivalently, we can parametrize

$$
\Gamma_{\mu}^{(1)}(t)=t \delta_{\mu 3}, \quad \Gamma_{\mu}^{(2)}(l)=-l \delta_{\mu 2}, \quad \Gamma_{\mu}^{(3)}(t)=-t \delta_{\mu 3}, \quad \Gamma_{\mu}^{(4)}(l)=l \delta_{\mu 2},
$$

with $t \in[-T, T]$ and $l \in[-L, L]$. The Wilson loop can be easily computed by expanding in powers of the coupling constant,

$$
W_{\Gamma_{L T}}=W_{\Gamma_{L T}}^{(0)}+g W_{\Gamma_{L T}}^{(1)}+\frac{g^{2}}{2} W_{\Gamma_{L T}}^{(2)}+\frac{g^{3}}{3 !} W_{\Gamma_{L T}}^{(3)}+O\left(g^{4}\right) .
$$

By using the properties of the generators in the fundamental representation of $S U\left(N_{c}\right)$, $\operatorname{Tr} 1=N_{c}$, $\operatorname{Tr} T_{a}=0$ and $\operatorname{Tr}\left(T_{a} T_{b}\right)=\frac{1}{2} \delta_{a b}$, from (99) one obtains the explicit expression

$$
W_{\Gamma_{L T}}=1-\frac{g^{2}}{4 N_{c}} \int_{\Gamma_{L T}} d x^{\mu} d y^{\nu}<A_{\mu}(x) \cdot A_{\nu}(y)>^{(0)}+O\left(g^{4}\right) .
$$

Notice that higher orders corrections begins at order $O\left(g^{4}\right)$, not $O\left(g^{3}\right)$. The expectation value $<A_{\mu}(x) \cdot A_{\nu}(y)>^{(0)}$, computed at zero order in the coupling constant, coincides with the Fourier transform of the free propagator, therefore a a more explicit expression for the $O\left(g^{2}\right)$ contribution is

$$
W_{\Gamma_{L T}}^{(2)}=-C_{F} \int_{-1}^{1} d s_{1} \int_{-1}^{1} d s_{2} \frac{\mathrm{d} \Gamma^{\mu}}{\mathrm{d} s_{1}} \frac{\mathrm{d} \Gamma^{\nu}}{\mathrm{d} s_{2}} D_{\mu \nu}\left(\Gamma\left(s_{1}\right)-\Gamma\left(s_{2}\right)\right),
$$

where the relation $\delta^{a a}=2 N_{c} C_{F}$ with $C_{F}=\left(N_{c}^{2}-1\right) /\left(2 N_{c}\right)$ has been used. Specializing to the rectangular Wilson loop in figure 2 we have in general sixteen contribution to (105); however many terms gives the same contribution 
due to the symmetries $D_{\mu \nu}(x)=D_{\nu \mu}(x)$ and $D_{\mu \nu}(x)=D_{\mu \nu}(-x)$ and finally one is left with the explicit expression

$$
\begin{array}{r}
\frac{W_{\Gamma_{L T}}^{(2)}=-}{2 C_{F}}=\int_{-T}^{T} d t_{1} \int_{-T}^{T} d t_{2}\left[D_{33}\left(0,2 L, t_{1}+t_{2}, 0\right)+D_{33}\left(0,0, t_{1}-t_{2}, 0\right)\right] \\
-\int_{-L}^{L} d l_{1} \int_{-L}^{L} d l_{2}\left[D_{22}\left(0, l_{1}+l_{2}, 2 T, 0\right)+D_{22}\left(0, l_{1}-l_{2}, 0,0\right)\right] \\
+4 \int_{-T}^{T} d t \int_{-L}^{L} d l D_{23}(0, L+l, T-t, 0) .
\end{array}
$$

This formula can be further simplified in the $T \rightarrow \infty$ limit since various contributions are subleading. Moreover, due to the identity (which holds since $\Gamma_{L T}$ is a closed path)

$$
\int_{\Gamma_{L T}} d x^{\mu} d y^{\nu} D_{\mu \nu}(x-y)=\int_{\Gamma_{L T}} d x^{\mu} d y^{\nu} D_{\mu \nu}^{T}(x-y),
$$

actually only the transverse part of the propagator contributes. At zero mass $D_{\mu \nu}^{T}(p)$ is the same in all gauges and this is the reason why the final result is gauge-independent; however at $\Lambda \neq 0$ there is a dependence on the gauge vector $n_{\mu}$ coming from the $\tilde{D}_{\Lambda, \mu \nu}$ part of the propagator.

A naive expectation would suggest that $\tilde{D}_{\Lambda, \mu \nu}$ being proportional to $\Lambda^{2}$ gives a vanishing contribution to the Wilson loop. This is in general should not be taken for granted. Nevertheless, suppose for a moment that this naive espectation is correct (this point will be analyzed in detail in next section). Then one can consider only the $\bar{D}_{\mu \nu}$ part of the propagator or even only the transverse part $\bar{D}_{\mu \nu}^{T}$. This object is independent of the gauge-fixing vector $n_{\mu}$ and therefore the Wilson loop is the same as in covariant gauges. In particular, we can effectively replace $\bar{D}_{\mu \nu}$ with $\delta_{\mu \nu} /\left(p^{2}+\Lambda^{2}\right) \equiv \delta_{\mu \nu} D_{\Lambda}(p)$ because they have the same transverse part. If we divide by $2 T$ in order to eliminate the contributions subleading at $T \rightarrow \infty$ we obtain for the $O\left(g^{2}\right)$ analogous of the interquark potential

$$
V_{\Lambda}^{(2)}(2 L) \equiv-\lim _{T \rightarrow \infty} \frac{g^{2} N_{c} W_{\Gamma_{L T}}^{(2)}}{4 T}
$$

the expression

$$
V_{\Lambda}^{(2)}(2 L)=\frac{g^{2} N_{c} C_{F}}{2 T} \int_{-T}^{T} d t_{1} \int_{-T}^{T} d t_{2} D_{\Lambda}\left(t_{1}+t_{2}, 2 L\right)+c
$$


where $c$ is the infinite renormalization constant

$$
c=\lim _{T \rightarrow \infty} \frac{g^{2} N_{c} C_{F}}{2 T} \int_{-T}^{T} d t_{1} \int_{-T}^{T} d t_{2} D_{\Lambda}\left(t_{1}-t_{2}, 0\right) .
$$

This is the well known ultraviolet divergence of point-like charges and can be eliminated by fixing the potential to be zero at $L \rightarrow \infty$. With simple manipulations involving the representation of the delta function

$$
2 \pi \delta\left(p_{3}\right)=\lim _{T \rightarrow \infty} \int_{-T}^{T} d x_{3} e^{i p_{3} x_{3}}=\lim _{T \rightarrow \infty} \frac{2 \sin \left(p_{3} T\right)}{p_{3}}
$$

and the formula

$$
\lim _{T \rightarrow \infty} \frac{\left(2 \sin p_{3} T\right)^{2}}{p_{3}^{2}}=\lim _{T \rightarrow \infty} 2 T \cdot 2 \pi \delta\left(p_{3}\right),
$$

from $(109)$ one obtains

$$
V_{\Lambda}^{(2)}(2 L)=g^{2} N_{c} C_{F} \int_{\bar{p}} \frac{\exp \left(i p_{2} \cdot 2 L\right)}{p^{2}+\Lambda^{2}}=g^{2} N_{c} C_{F} \frac{\exp (-\Lambda \cdot 2 L)}{4 \pi \cdot 2 L} .
$$

This is the formula of the screened Coulomb potential of two colored charges at distance $2 L$ and reduces to the standard one when the infrared cutoff is removed. We still stress that this formula is correct as far as we can neglect the contribution from the $\tilde{D}_{\Lambda}$ term in the limit $\Lambda \rightarrow 0$.

\subsection{Vanishing of the $\tilde{D}_{\Lambda}$ term}

Now we prove that the $\tilde{D}_{\Lambda}$ term of the propagator gives a vanishing contribution to the Wilson loop. This is a non-trivial point since in this quantity there is a problem of commutativity between the limits $\Lambda \rightarrow 0$ and $T \rightarrow \infty$. This can be seen from the explicit expression of the $\tilde{D}_{\Lambda}$ contribution to the interquark potential, which up to an unessential infinite constant reads, working for definiteness in the planar gauge,

$$
\begin{aligned}
\tilde{V}_{\Lambda}^{(2)}(2 L) & =\frac{g^{2} N_{c} C_{F}}{2 T} \int_{-T}^{T} d t_{1} \int_{-T}^{T} d t_{2} D_{33}\left(0,2 L, t_{1}+t_{2}, 0\right) \\
= & -\frac{g^{2} N_{c} C_{F}}{2 T} \int_{p_{E}} \frac{e^{i p_{2} \cdot 2 L} \Lambda^{2}}{\left[p_{3}\right]^{2}\left(p_{3}^{2}+\bar{p}^{2}+\Lambda^{2}\right)} \frac{\left(2 \sin p_{3} T\right)^{2}}{p_{3}^{2}} .
\end{aligned}
$$


We see that we cannot blindly use the formula (112) and compute the integral directly at $T \rightarrow \infty$. We are instead forced to consider finite $T$ and to make some subtle observation. The idea is that the $p_{3}$-integral is dominated by the $p_{3} \simeq 0$ region and that actually it reduces to an integral of the kind

$$
I_{\alpha}(T)=\int_{p_{3}} \frac{\left(2 \sin p_{3} T\right)^{2}}{\left(p_{3}\right)^{2+2 \alpha}}
$$

with $\alpha=1$. Strictly speaking this is a divergent integral; nevertheless it can be defined in the region $-1 / 2<\alpha<1 / 2$ where its value is

$$
I_{\alpha}(T)=\frac{2}{\pi} \Gamma(-1-2 \alpha) \sin (\alpha \pi)(2 T)^{1+2 \alpha} .
$$

After analytic continuation to $\alpha=1$ we obtain

$$
I_{1}(T)=-\frac{4}{3} T^{3}
$$

A simpler way to derive this formula is to derive equation (117) three times with respect to $T$ : then the sinus representation of the delta function is recovered and the integral is easily computed. In other words we can use the formula, which has to be interpreted in the sense of distributions,

$$
\lim _{T \rightarrow \infty} \frac{\left(2 \sin p_{3} T\right)^{2}}{\left[p_{3}\right]^{4}}=\lim _{T \rightarrow \infty}-\frac{4}{3} T^{3} \cdot 2 \pi \delta\left(p_{3}\right) .
$$

Then the three-dimensional integration is as in (113) and the final result is

$$
\tilde{V}_{\Lambda}^{(2)}(2 L)=\frac{2 g^{2} N_{c} C_{F}}{3} \Lambda^{2} T^{2} \frac{\exp (-\Lambda \cdot 2 L)}{4 \pi \cdot 2 L} .
$$

We see that this contribution is quadratically vanishing in the limit $\Lambda \rightarrow 0$, at finite $T$. The $T \rightarrow \infty$ limit cannot be taken before the $\Lambda \rightarrow 0$ limit. This is the crucial point of our analysis.

For what concerns the situation with other gauges, we observe that in the light-cone gauge we have to compute exactly the same integral as in (114) and therefore we have the same result. On the contrary, in the massive axial gauge we have to replace a factor $1 /\left[p_{3}\right]^{2}$ with a factor $\left(p_{3}^{2}+\Lambda^{2}\right)^{-1}$ and using (48) we see that the final result is linearly vanishing with $\Lambda T$. This means that the $O\left(g^{2}\right)$ Wilson loop test works for any gauge choice: but this is hardly 
a surprise since the $O\left(g^{2}\right)$ computation corresponds to the computation in an Abelian theory. It is well known that problems begin in the non-Abelian case and at higher orders in perturbation theory, starting from order $O\left(g^{4}\right)$, therefore we cannot conclude nothing about the final consistency of these Wilsonian gauge choices at this level. Nevertheless we think that this analysis of the free case is very instructive and allows to learn a lot about the possible origin of problems. In particular we have learned that in an $O\left(g^{4}\right)$ computation the only part of the propagator we have to control is the $\tilde{D}_{\Lambda}$ part, which is quite simple and surely can be studied with a relatively little analitical effort. In other Wilsonian formulations of non-covariant gauges based on infrared cutoffs more complicate than a mass-like term [16, 17] the analysis is technically much more cumbersome but physically equivalent in what concerns the final results in the $\Lambda \rightarrow 0$ limit. The drawback of the generic cutoff is that the fine-tuning problem must be solved in order to fix the correct boundary conditions (renormalization prescriptions) such to have an infrared limit consistent with the gauge-symmetry; this difficult problem is avoided with the mass cutoff since with this choice the theory is Ward-identities-consistent to all scales. Finally we would stress the fact the planar gauge and in particular the light-cone gauge are expected to be much more regular than the axial gauge in the zero mass limit: this expectation is reflected in the present computation by the fact that the $\tilde{D}_{\Lambda}$ term

is quadratically suppressed in both planar and light-cone gauge and merely linearly suppressed in axial gauge.

\section{Conclusions}

In 99 we pointed out that in algebraic non-covariant gauges it is possible to build up a Wilsonian formulation of gauge theories consistent with the Ward identities provided that the infrared cutoff is introduced as a formal "mass" term. However, we stressed that this infrared cutoff cannot be physically interpreted and must be removed in order to recover the essential property of gauge-independence of physical quantities. In this paper we have investigated the properties of the singular limit $\Lambda \rightarrow 0$ by explicitly computing various quantities. We have seen in general that the pure axial gauge choice is problematic since the Fourier transform of the propagator and even the simplest Feynman diagrams which are finite in covariant gauges are instead divergent at $\Lambda \rightarrow 0$, for any configuration of momenta. Moreover, we argued 
that other gauge choices, namely the planar gauge and the light-cone gauge are in a much better shape. We have however seen that the structure of infrared divergences is quite subtle even in these cases and that there are gauge-dependent quantities such as for on-shell configurations of momenta the contribution from the gauge-dependent term of the propagator $\tilde{D}_{\Lambda, \mu \nu}$ is not only non-vanishing, but even it is dominant with respect to the standard contribution. Therefore the $\Lambda \rightarrow 0$ limit for these quantities is delicate. However these quantities are unphysical and this fact should not be considered as suggesting an inconsistency of the theory. The only way to prove the consistency or the inconsistency of the approach is by considering true physical quantities like the interquark potential as obtained from a Wilson loop of size $2 L \times 2 T$ with $T \rightarrow \infty$. We have seen that the limits $\Lambda \rightarrow 0$ and $T \rightarrow \infty$ must be studied with great care, since they do not commute. In particular the crucial property of the exponentiation of the Wilson loop is recovered only if the $\Lambda \rightarrow 0$ limit is taken before the $T \rightarrow \infty$ limit. We have seen that the planar gauge and the light-cone gauge appear to be much more regular than the axial gauge in the infrared limit. This is explicitly realized in the Wilson loop $O\left(g^{2}\right)$ computation by the fact that the gauge dependent term is quadratically suppressed at small $\Lambda T$ whereas in axial gauge this term is only linearly suppressed. We notice that in covariant gauges, by accident, the order of limits is not crucial at order $O\left(g^{2}\right)$, then this feature has not been recognized previously; nevertheless it is manifest in an $O\left(g^{4}\right)$ computation. The reason is that the infrared cutoff breaks the BRST-invariance and therefore the cancellation of the so called non-Abelian contributions to the Wilson loop no more works at $\Lambda \neq 0$. Therefore the interquark potential cannot be perturbatively defined at $\Lambda \neq 0$ and $T \rightarrow \infty$, but only at finite $T$. Finally we notice that the physical interquark potential, defined exactly at $\Lambda=0$, is independent of the cutoff function choice when it is computed order by order in perturbation theory.

For the future, we plan to study in detail what happens in the $O\left(g^{4}\right)$ computation. Actually, at finite $T$, we expect that in planar and light-cone gauges the computation reduces smoothly to the standard massless computation, which is consistent, whereas the axial gauge should be very delicate and possibly inconsistent. Still, we should notice that even in the cases where the $O\left(g^{4}\right)$ Wilson loop test fails, in principle this only indicate a failure of the perturbative expansion and not necessarily of the full theory. Nevertheless one would feel much more confortable with an approach admitting a perturbative expansion. In such a perspective, the most promising possibility seems 
to be the light-cone gauge which certainly deserves additional investigations.

Note Added. During the completion of this work we received a communication from R. Soldati and A. Panza [29] who explicitly computed the Wilson loop at order $O\left(g^{4}\right)$ in the massive axial gauge case and proved that the $\Lambda \rightarrow 0$ limit is singular. Therefore the axial gauge choice seems to be definitely pathological even in the Wilson renormalization group approach, at least at the perturbative level.

\section{Acknowledgements}

I acknowledge support from Padova University during the early stages of this work. I thank R. Soldati and A. Panza for useful discussions and for communicating to me the result of [29] before publication.

\section{A Gauge-dependence of the pressure}

In this appendix we provide a very simple and illuminating example which illustrates the gauge-dependence problem in presence of a non-zero mass cutoff $\Lambda$.

Consider a free gas of photons in thermal equilibrium at temperature $T=1 / \beta$ in a box of volume $V$. We can compute, as a typical quantity directly related to the partition function, the pressure of this gas in presence of the mass cutoff $\Lambda$. We obtain different result in different gauges, but all these results collapse to the correct physical result in the physical limit $\Lambda \rightarrow 0$. This is trivial example, since the theory is free, nevertheless we believe it is very instructive. We recall that the pressure is a typical quantity which cannot be computed in perturbation theory in thermal field theory and where the non-perturbative powerfulness of the Wilson renormalization group approach could give an alternative way of facing the problem. In this sense it is an interesting quantity.

We begin the computation by recalling some elementary facts (see for instance [22]). In quantum field theory the thermodynamic pressure

$$
p(\beta, V)=\frac{1}{\beta V} \ln Z_{\beta V}
$$


is defined in terms of the partition function

$$
Z_{\beta V}=\int[d \phi] \exp \left(-\int_{0}^{\beta} d \tau \int_{V} d^{3} x \mathcal{L}_{E}\left(\phi, \partial_{\mu} \phi ; \Lambda_{0}\right)\right)
$$

where $\mathcal{L}_{E}\left(\phi, \partial_{\mu} \phi ; \Lambda_{0}\right)$ is the bare Euclidean lagrangian of the theory, which is a function of the bare parameters depending on the ultraviolet cutoff $\Lambda_{0}$. For instance for a free scalar field of mass $\Lambda$ we have,

$$
\mathcal{L}_{E}\left(\phi, \partial_{\mu} \phi ; \Lambda_{0}\right)=\left(\frac{1}{2} \partial_{\mu} \phi \partial^{\mu} \phi\right)_{\Lambda_{0}}+\frac{1}{2} \Lambda^{2} \phi^{2}+c_{4}\left(\Lambda, \Lambda_{0}\right)
$$

where the notation $\left(\frac{1}{2} \partial_{\mu} \phi \partial^{\mu} \phi\right)_{\Lambda_{0}}$ reminds that there is an ultraviolet cutoff inserted in the propagator in momentum space and the term $c_{4}\left(\Lambda, \Lambda_{0}\right)=$ $\Lambda_{0}^{4}\left[\tilde{c}_{4}+\tilde{c}_{4}^{\prime} \Lambda^{2} / \Lambda_{0}^{2}+O\left(\Lambda^{4} / \Lambda_{0}^{4}\right)\right]$ is a vacuum energy counterterm of dimension four, which will be fixed later on by imposing the normalization condition

$$
\lim _{\beta \rightarrow \infty} \ln Z_{\beta V}=0
$$

i.e. the partition function is fixed to be 1 at zero temperature. It is interesting to notice that in order to impose this normalization the ultraviolet regularization is needed even if the theory is free. Using the gaussian integration formula we obtain

$$
Z_{\beta V}=\mathcal{N} \operatorname{det}_{\beta V}\left(-\partial_{E}^{2}+\Lambda^{2}\right)^{-1 / 2} \exp \left(-c_{4}\left(\Lambda, \Lambda_{0}\right) \beta V\right)
$$

where $\mathcal{N}$ is a temperature independent normalization factor to be fixed later whereas the determinant of the operator $-\partial_{E}^{2}+\Lambda^{2}$ (acting on functions periodic in the imaginary time) is defined as

$$
\operatorname{det}_{\beta V}\left(-\partial_{E}^{2}+\Lambda^{2}\right)^{-1 / 2} \equiv \exp \left[-\frac{1}{2} \operatorname{Tr}_{\beta V} \ln \left(-\partial_{E}^{2}+\Lambda^{2}\right)\right]
$$

with

$$
\operatorname{Tr}_{\beta V} \ln \left(-\partial_{E}^{2}+\Lambda^{2}\right) \equiv V \sum_{n=-\infty}^{\infty} \int_{\vec{p}} \ln \left[\left(p_{n}^{4}\right)^{2}+\vec{p}^{2}+\Lambda^{2}\right], \quad p_{n}^{4} \equiv 2 \pi n T .
$$


Using the stardard summation formula]

$$
\sum_{n=-\infty}^{\infty} \ln \left[\left(p_{n}^{4}\right)^{2}+X^{2}\right]=-2 \log [1+n(X)]+\beta X
$$

where $n(X)=(\exp (\beta X)-1)^{-1}$ is the Bose-Einstein distribution function, one obtains

$$
\ln Z_{\beta V}=V \int_{\vec{p}} \ln \left[1+n\left(\omega_{p}(\Lambda)\right)\right]-\frac{1}{2} \beta V \int_{\vec{p}}^{\Lambda_{0}} \omega_{p}(\Lambda)-\beta V c_{4}+\ln \mathcal{N},
$$

with $\omega_{p}(\Lambda)=\sqrt{\vec{p}^{2}+\Lambda^{2}}$. The first term vanishes at $\beta \rightarrow \infty$. Imposing the normalization prescription (123) fixes the counterterm $c_{4}\left(\Lambda, \Lambda_{0}\right)$ and the normalization factor $\mathcal{N}$ to be

$$
c_{4}\left(\Lambda, \Lambda_{0}\right)=-\frac{1}{2} \int_{\vec{p}}^{\Lambda_{0}} \omega_{p}(\Lambda), \quad \mathcal{N}=1
$$

therefore the pressure reduces to the well known expression

$$
p(T, \Lambda)=T \int_{\vec{p}} \ln \left[1+n\left(\omega_{p}\right)\right] \stackrel{\Lambda \rightarrow 0}{=} \frac{\pi^{2}}{90} T^{4} .
$$

Now we can repeat the same computation in the gauge theory case which is less trivial. We will consider the general linear gauge (16) with $\xi_{2} \neq 0$. Using the Gaussian integration formula we obtain

$$
Z_{\beta V}\left(L, \xi_{2}\right)=\mathcal{N} \operatorname{det} \xi_{2}^{-1 / 2} \operatorname{det}_{\beta V} \partial^{\mu} L_{\mu} \operatorname{det}_{\beta V} D_{\mu \nu}^{1 / 2}
$$

where $\operatorname{det} \xi_{2}^{-1 / 2}$ comes from the integration of auxiliary fields, $\operatorname{det}_{\beta V} \partial^{\mu} L_{\mu}$ from the integration of ghost fields and $\operatorname{det}_{\beta V} D_{\mu \nu}^{1 / 2}$ from the integration of gauge fields. With a lengthy but straighforward computation, one obtains the determinant of the Euclidean propagator in the general class of linear gauges as

$$
\operatorname{det} D_{\Lambda, \mu \nu}(p)=\frac{1}{\left(p^{2}+\Lambda^{2}\right)^{2}} \frac{\xi_{2}}{(p \cdot L)^{2}+\Lambda^{2}\left[L^{2}+\xi_{2}\left(p^{2}+\Lambda^{2}\right)\right]} .
$$

\footnotetext{
${ }^{7}$ In order to use equation (127) we have to remove the ultraviolet cutoff in the energy variable $p_{4}$, otherwise the summation reduces to a finite sum with $N \sim \Lambda_{0} / T$ terms. In general the summation formula is defined up to a possibly divergent constant which can be reabsorved in the vacuum energy counterterm $c_{4}\left(\Lambda, \Lambda_{0}\right)$.
} 
Suppose for a moment that $\Lambda=0$ : in this case we see that the partition function is gauge-independent since the $\xi_{2}$ term in the photon propagator determinant is cancelled by the contribution coming from the integration of the auxiliary fields whereas the $L$-dependent part is cancelled by an analogous contribution from the ghost propagation, which in turn is fixed by requiring BRST-invariance of the total action. However, as can be explicitly seen, this cancellation mechanism of the gauge-dependence does not work in presence of an infrared regulator which breaks BRST-invariance. Therefore at finite $\Lambda$ one obtains an unphysical dependence on the gauge-fixing parameters. In particular in the limit $\xi_{2} \rightarrow 0$ i.e. strictly imposing the condition $L^{\mu} A_{\mu}=0$ one obtains for the logarithm of the partition function the explicit expression

$$
\begin{aligned}
\ln Z_{\beta V} & =2 \operatorname{Tr}_{\beta V} \ln \left(-\partial_{E}^{2}+\Lambda^{2}\right)^{-1 / 2}+\operatorname{Tr}_{\beta V} \ln \left((\partial \cdot L)^{2}+L^{2} \Lambda^{2}\right)^{-1 / 2} \\
& -\operatorname{Tr}_{\beta V} \ln \left((\partial \cdot L)^{2}\right)^{-1 / 2}-\beta V c_{4}\left(\Lambda, \Lambda_{0}\right)+\ln \mathcal{N} .
\end{aligned}
$$

The first term is the expected one, corresponding to the pressure of two bosonic degrees of freedom; however there are also terms which are explicitly $L$-dependent at $\Lambda \neq 0$ and therefore unphysical; the $L$-dependence only cancels at $\Lambda \rightarrow 0$ where one recovers the correct result

$$
p(T ; L) \stackrel{\Lambda \rightarrow 0}{=} \frac{\pi^{2}}{45} T^{4} \quad \forall L
$$

This is the generic situation, however it is interesting to study what happens in specific gauge choices since the gauge-dependence of the pressure can be less prononced than expected. For instance in the light-cone gauge $L^{2}=n^{2}=0$ we see that the $n_{\mu}$-dependent terms cancel even at $\Lambda \neq 0$. Actually the $n_{\mu}$-dependence cancels in the large class of non-covariant gauges such as $L_{\mu}(p)$ does not depend on the $p_{4}$ variable (for instance this is the case for the planar gauge and the Coulomb gauge): in this case the $L$-dependent terms in $\ln Z_{\beta V}$ have the form $\beta V f\left(L, \Lambda, \Lambda_{0}\right)$ and therefore can be reabsorved in the in the vacuum energy counterterm $c_{4}\left(\Lambda, \Lambda_{0}\right)$. In other words, they are eliminated by the normalization condition (123) and do not contribute to the pressure even at $\Lambda \neq 0$. However the dependence on the quantization direction should be expected in a two-loop computation; moreover it is evident in other observables, for instance in the Wilson loop computation, therefore in any case the infrared cutoff $\Lambda$ cannot be physically interpreted as a true mass term, consistently with the standard lore that the only way to give a physical mass to a non-Abelian theory is via the Higgs mechanism. Nevertheless from 
this simple example one could extrapolate the conjecture (to be checked case by case perturbatively with higher order computations or non-perturbatively with a numerical analysis) that for particular observables there are classes of gauges more regular that others, in which the gauge-dependence is very mild even for non-zero $\Lambda$. Clearly, this is a very interesting point in the spirit of phenomenological numerical analysis and should be investigated in the future.

\section{B One-loop integrals}

In this appendix we give some generalities on the computation of Euclidean one-loop integrals in planar and light-cone gauges. We begin by fixing our notations on integrals: for axial and planar gauges we define

$$
\int_{x}=\int d^{4} x, \quad \int_{q}=\int \frac{d^{4} q}{(2 \pi)^{4}}, \quad \int_{q_{3}}=\int \frac{d q_{3}}{2 \pi}, \quad \int_{\bar{q}}=\int \frac{d^{3} \bar{q}}{(2 \pi)^{3}},
$$

with $\bar{q}=\left(q^{0}, q^{1}, q^{2}\right)$, whereas for the light-cone gauge we define

$$
\int_{q_{\perp}}=\int \frac{d^{2} q_{\perp}}{(2 \pi)^{2}}, \quad \int_{q_{\|}}=\int \frac{d^{2} q_{\|}}{(2 \pi)^{2}}
$$

with $\vec{q}_{\perp}=\left(q^{1}, q^{2}\right), \vec{q}_{\|}=\left(q^{3}, q^{0}\right)$. Euclidean vectors are obtained after Wick rotation $p_{4}=i p_{0}$; we shall use the notations

$$
p_{E}=\left(\vec{p}, i p_{0}\right), \quad q_{E}=\left(\vec{q}, i q_{0}\right), \quad p_{E} q_{E} \equiv \delta_{\mu \nu} p_{E}^{\mu} q_{E}^{\nu}=-g_{\mu \nu} p^{\mu} q^{\nu}=-p q
$$

and

$$
\begin{gathered}
p_{\|}^{2}=p_{3}^{2}, \quad p_{\perp}^{2}=\bar{p}^{2} \quad \text { (planar gauge) } \\
p_{\|}^{2}=p_{3}^{2}+p_{4}^{2}, \quad p_{\perp}^{2}=p_{1}^{2}+p_{2}^{2} \quad \text { (light-cone gauge) }
\end{gathered}
$$

In this appendix we will always work in Euclidean space even if for sake of notational convenience the index $E$ will be neglected.

For semplicity we will restrict our remarks to the computation of the one-loop self-energy of a scalar quark. In general this is a rather complicate

function $F=F\left(p_{\|}, p_{\perp}, \Lambda\right)$, but we can give analytical estimations in the two 
limits $\left|p_{\|}^{2}\right| \ll p_{\perp}^{2}$ and $\left|p_{\|}^{2}\right| \gg p_{\perp}^{2}$. In the first case by putting $p_{\|}=0$ we see that the spurious terms $1 /[q \cdot n]$ cancel and then the one-loop integral can be put in an explicitly covariant form: therefore it can be computed through the usual Feynman parametrization. The case $\left|p_{\|}^{2}\right| \gg p_{\perp}^{2}$ instead is more cumbersome, nevertheless it can be easily implemented with a symbolic manipolation package. As a matter of fact, we prepared a set of routines based on the double Feynman parametrization method allowing to compute analytically all the integrals which are encountered in one-loop self-energy diagrams, including finite parts, and we checked that the $\Lambda \rightarrow 0$ limit reproduces the known results of the standard approach. However, for sake of brevity, here we simply sketch the analysis for the simplest examples: the generalization to more complicate cases is straighforward.

The general form of the Feynman integral one encounters in the evaluation of the quark self-energy (or its derivatives with respect to the mass) is given by the expression

$$
F\left((p \cdot n)^{2}, p^{2}, \Lambda\right)=\int_{q} \frac{N(q, p, n)}{[q \cdot n]\left(q^{2}+\Lambda^{2}\right)\left((q+p)^{2}+m^{2}+\Lambda^{2}\right)^{1+\alpha}} .
$$

The integral (140) will be explicitly evaluated both in planar gauge and lightcone gauge in next subsections.

\section{B.1 Planar gauge integrals}

Consider first the planar gauge case where $q \cdot n=q_{3}$. The most convenient technical tool we found to manage this kind of integrals is the double Feynman parametrization which consists in using the identities

$$
\frac{1}{q_{3}} \frac{1}{q^{2}+\Lambda^{2}}=\int_{0}^{1} d y \frac{q_{3}}{\left[q_{3}^{2}+y\left(\bar{q}^{2}+\Lambda^{2}\right)\right]^{2}}
$$

and

$$
\begin{aligned}
& \int_{q} \frac{N(q, p, n)}{\left(q^{2}+A^{2}\right)^{a}\left((q+p)^{2}+m^{2}+\Lambda^{2}\right)^{b}}= \\
& \frac{1}{B(a, b)} \int_{q} \int_{0}^{1} d x \frac{N(q-p(1-x), p, n) x^{a-1}(1-x)^{b-1}}{\left[q_{3}^{2}+A x+\left(\bar{q}^{2}+p^{2} x+m^{2}+\Lambda^{2}\right)(1-x)\right]^{a+b} .}
\end{aligned}
$$

Then it is convenient to introduce the variable $z=(y-1) x+1$ lying in the interval $1-x \leq z \leq 1$ and to rescale $\tilde{q}=z^{1 / 2} \bar{q}$; in this way equation (140) 
can be rewritten in the form

$$
F=\int_{q_{3}, \tilde{q}} \int_{0}^{1} d x \int_{1-x}^{1} d z \frac{\left[q_{3} N(q-p(1-x), p, n)\right]_{t r}(1-x)^{\alpha}}{z^{3 / 2}\left[q_{3}^{2}+\tilde{q}^{2}+\left(p^{2} x+m^{2}\right)(1-x)+\Lambda^{2} z\right]^{3+\alpha}}
$$

where we have introduced the translated and rescaled numerator

$$
\left[q_{3} N(q, p, n)\right]_{t r}=\left[q_{3} N(q, p, n)\right]_{q_{3} \rightarrow q_{3}-p_{3}(1-x)}^{\bar{q}^{2}=\tilde{q}^{2} / z} .
$$

After symmetrization in $q_{3}$ and three-dimensional angular average in the numerator, the momentum integrals can be performed by using the general formula

$$
\int_{q_{3}, \tilde{q}} \frac{\left(q_{3}^{2}\right)^{M_{1}}\left(\tilde{q}^{2}\right)^{M_{2}}}{\left(q_{3}^{2}+\tilde{q}^{2}+A\right)^{N}}=\frac{B_{1}\left(M_{1}, M_{2}, N\right)}{8 \pi^{3} A^{N-M_{1}-M_{2}-2}}
$$

where $B_{1}\left(M_{1}, M_{2}, N\right)$ denotes the product of beta functions

$$
B_{1}=B\left(N-M_{1}-M_{2}-2, M_{2}+3 / 2\right) B\left(N-M_{1}-1 / 2, M_{1}+1 / 2\right) .
$$

The integral in $z$ is trivial at $\Lambda=0$ and a little complicate at $\Lambda \neq 0$ but still expressible in terms of elementary functions; the integral in $x$ instead in non-trivial. Nevertheless one can explicitly check that it is finite and in general can be expressed in terms of special functions. In the $\Lambda \rightarrow 0$ limit the analysis strongly simplifies and one obtain the esplicit result reported in the text.

With a simple generalization of this method one can compute Feynman integrals where the double pole $1 /[q \cdot n]^{2}$ appears, without encountering any problem. Moreover, the extension of this method to the integrals appearing in the gluon self-energy computation is straightforward.

\section{B.2 Light-cone gauge integrals}

Consider now the light-cone gauge case where $q \cdot n=i q_{4}+q_{3}$. Still the double Feynman parametrization can be used, it is enough to replace identity (141) with

$$
\frac{1}{i q_{4}+q_{3}} \frac{1}{q^{2}+\Lambda^{2}}=\int_{0}^{1} d y \frac{-i q_{4}+q_{3}}{\left[q_{4}^{2}+q_{3}^{2}+y\left(q_{\perp}^{2}+\Lambda^{2}\right)\right]^{2}}
$$


Using (142), introducing the variable $z=(y-1) x+1$ and rescaling $\tilde{q}_{\perp}=$ $z^{1 / 2} q_{\perp}$ the Feynman integral (140) can be rewritten in the form

$$
F=\int_{q_{\|}, \tilde{q}_{\perp}} \int_{0}^{1} d x \int_{1-x}^{1} d z \frac{\left[\left(-i q_{4}+q_{3}\right) N(q, p, n)\right]_{t r}(1-x)^{\alpha}}{z\left[q_{\|}^{2}+\tilde{q}_{\perp}^{2}+\left(p^{2} x+m^{2}\right)(1-x)+\Lambda^{2} z\right]^{3+\alpha}}
$$

where we have defined the translated and rescaled numerator as

$$
\left[\left(-i q_{4}+q_{3}\right) N(q, p, n)\right]_{t r}=\left[\left(-i q_{4}+q_{3}\right) N(q, p, n)\right]_{\vec{q}_{\|} \rightarrow \vec{q}_{\|}-\vec{p}_{\|}(1-x)}^{q_{2}^{2}=\tilde{q}_{\perp}^{2} / z} .
$$

Now we can perform the angular average in two dimensions

$$
<f\left(\vec{q}_{\|} \cdot \vec{p}_{\|}\right)>_{\Omega_{2}} \equiv \frac{1}{2 \pi} \int_{0}^{2 \pi} d \theta f\left(q_{\|} p_{\|} \cos \theta\right),
$$

by using the general formulae

$$
<\left(\vec{q}_{\|} \cdot \vec{p}_{\|}\right)^{2 n}>_{\Omega_{2}}=\frac{\Gamma(n+1 / 2)}{\sqrt{\pi} \Gamma(n+1)} q_{\|}^{2 n} p_{\|}^{2 n}, \quad<\left(\vec{q}_{\|} \cdot \vec{p}_{\|}\right)^{2 n+1}>_{\Omega_{2}}=0
$$

In particular

$$
<\left(\vec{q}_{\|} \cdot \vec{p}_{\|}\right)^{2}>_{\Omega_{2}}=\frac{1}{2} q_{\|}^{2} p_{\|}^{2}, \quad<\left(\vec{q}_{\|} \cdot \vec{p}_{\|}\right)^{4}>_{\Omega_{2}}=\frac{3}{8} q_{\|}^{4} p_{\|}^{4} .
$$

The integrals in $q_{\|}, \tilde{q}_{\perp}$ can be performed by using the general formula

$$
\int_{q_{\|}, \tilde{q}_{\perp}} \frac{\left(q_{\|}^{2}\right)^{M_{1}}\left(\tilde{q}_{\perp}^{2}\right)^{M_{2}}}{\left(q_{\|}^{2}+\tilde{q}_{\perp}^{2}+A\right)^{N}}=\frac{B_{2}\left(M_{1}, M_{2}, N\right)}{16 \pi^{2} A^{N-M_{1}-M_{2}-2}}
$$

where

$$
B_{2}=B\left(N-M_{1}-M_{2}-2, M_{2}+1\right) B\left(N-M_{1}-1, M_{1}+1\right) .
$$

The integral in $z$ is is trivial at $\Lambda=0$ and a little complicate at $\Lambda \neq 0$ but still expressible in terms of $\operatorname{logarithms~of~} \log (1-x)$; the integral in $x$ in nontrivial. Nevertheless one can explicitly check that it is finite and in general can be expressed in terms of polylogarithmic functions. In the $\Lambda \rightarrow 0$ limit and in the on-shell regime $p^{2} \rightarrow-m^{2}$ the full expression strongly simplifies and we obtain the expression involving logarithms given in the text.

With a simple generalization of this method one can compute Feynman integrals where the double pole appears, without encountering any problem.

Moreover, the extension of this method to the integrals appearing in the gluon self-energy computation is straightforward. 


\section{B.3 Feynman parameters integrals}

Finally we report some useful trick to perform integrals on Feynman param-

eters in particular limits. Suppose one has to compute the integral of some function $f(x, \Lambda)$ which in the $\Lambda \rightarrow 0$ limit is dominate from the $x \simeq 0$ region; this is the case for instance for

$$
f(x, \Lambda)=\frac{P^{\nu}(x)}{\left(\Lambda^{2}+p^{2} x\right)^{n}}
$$

where $P^{\nu}(x)$ is a polynomial a degree $\nu$ in $x$, with $\nu<n-1$. This is the typical expression we encounter in the computation of self-energy diagrams; then one can use the identity

$$
\int_{0}^{1} d x f(x, \Lambda)=\int_{0}^{\infty} d x f(x, \Lambda)-\int_{1}^{\infty} d x f(x, \Lambda)
$$

and neglect the second contribution which is subleading at $\Lambda \rightarrow 0$. Then the first integral can be performed by using the formula

$$
\int_{0}^{\infty} d x \frac{x^{m}}{\left(\Lambda^{2}+p^{2} x\right)^{n}}=\frac{B(m+1, n-m-1)}{\Lambda^{2 n}}\left(\frac{\Lambda^{2}}{p^{2}}\right)^{m+1}
$$

which holds for $m>-1$ and $n>m+1$. In the case in which the integral is dominate by the $x \simeq 1$ region it is sufficient to change the variable $x^{\prime}=1-x$ and use the same trick.

\section{References}

[1] K.G. Wilson and I.G. Kogut, Phys. Rep. 12 (1974) 75

[2] C. Bagnuls, C. Bervillier, "Exact Renormalization Group Equations. An Introductory Review", hep-th/0002034

[3] C. Wetterich, Phys. Lett. 301B (1993) 90

[4] M.Bonini, M. D’Attanasio, G. Marchesini, Nucl. Phys. B409 (1993) 441

[5] U. Ellwanger, Phys. Lett. 335B (1994) 364 
[6] C. Becchi, in Elementary Particles, Field Theory and Statistical Mechanics, Eds. M. Bonini, G. Marchesini and E. Onofri, Parma University 1993, hep-th/9607188; M.Bonini, M. D'Attanasio, G. Marchesini, Phys. Lett. B346 (1995) 87 ; M. D'Attanasio and T.R. Morris, Phys. Lett. 378B (1996) 213

[7] M.Bonini, M. D’Attanasio, G. Marchesini, Nucl. Phys. B444 (1995) 602

[8] M. Simionato, "Gauge Consistent Wilson Renormalization Group I: Abelian case", hep-th/9809004, Int. J. Mod. Phys. A, Vol. 15, p. 2121 (2000)

[9] M. Simionato, "Gauge Consistent Wilson Renormalization Group II: Non-Abelian case", hep-th/9810117, Int. J. Mod. Phys. A, Vol. 15, p. 2153 (2000)

[10] N. Nakanishi, Prog. Theor. Phys. 67 (1982) 965

[11] G. Nardelli, R. Soldati, Int. J. Mod. Phys. A5 (1990) 3171

[12] U. Ellwanger, M. Hirsch and A. Weber, Z. Phys. 69 (1996) 687, Eur. Phys.J. C1 (1998) 563

[13] S. Caracciolo, G. Curci, P. Menotti, Phys. Lett. 113B (1982) 311

[14] J. P. Leroy, J. Micheli and G. C. Rossi, Nucl. Phys. B232, 511 (1984)

[15] A. Bassetto, G. Nardelli, R. Soldati, "Yang-Mills Theories in Algebraic Non-Covariant Gauges" (World Scientific, Singapore 1991)

[16] D. F. Litim, J. M. Pawlowski, Phys. Lett. 435B (1998) 181

[17] K. Geiger, Phys. Rev. D60 (1999) 034012

[18] A. Bassetto, R. Soldati, Nucl. Phys. B276 (1986) 517

[19] A. Bassetto, I. A. Korchemskaya, G. P. Korchemsky, G. Nardelli, Nucl. Phys. B408 (1993) 62

[20] G. Curci and R. Ferrari, Nuovo Cim. 32 (1976) 151; A. Blasi, N. Maggiore, Mod. Phys. Lett. A11, 1665-1674, 1996 
[21] T. Kugo, I. Ojima, "Covariant operator formalism of gauge theories", Suppl. of the Progress of Theoretical Physics 66 (1979)

[22] J. I. Kapusta, Finite Temperature Field Theory, Cambridge Monographs on Mathematical Physics, Cambridge Univ. Press, 1989.

[23] W. Kummer, Phys.Rev. D37, 454 (1988)

[24] O. Piguet and A. Rouet, Phys. Rep. 76 (1981) 1; O. Piguet and S. P. Sorella, "Algebraic renormalization", Lecture Notes in Physics, Vol. m28, Springer Verlag, Berlin, Heidelberg, 1995.

[25] G. Leibbrandt, "Introduction to Non-Covariant Gauges", Rev. Mod. Phys. 59 (1987) 1067; G. Leibbrandt, "Non-Covariant Gauges" (World Scientific, Singapore 1994)

[26] H. Cheng and E. C. Tsai, Phys. Rev. D 36 (1987) 3196

[27] S. D. Joglekar, A. Misra, Mod. Phys. Lett. A14: 2083-2092, 1999 and hep-th/9912020

[28] Y. Nakawaki, G. McCartor, "Indispensability of ghost fields and Extended Hamiltonian formalism in Axial Gauge Quantization of Gauge Fields", hep-th/0004140

[29] A. Panza, R. Soldati, "Infrared Singularities in the Renormalization Group Flow of Yang-Mills Theories in Axial Gauge", hep-th/0006170. 